\title{
Influence of trees and associated variables on soil organic carbon: a review
}

\author{
Angom Sarjubala Devi
}

\begin{abstract}
The level of soil organic carbon (SOC) fluctuates in different types of forest stands: this variation can be attributed to differences in tree species, and the variables associated with soil, climate, and topographical features. The present review evaluates the level of SOC in different types of forest stands to determine the factors responsible for the observed variation. Mixed stands have the highest amount of SOC, while coniferous (both deciduous-coniferous and evergreen-coniferous) stands have greater SOC concentrations than deciduous (broadleaved) and evergreen (broadleaved) tree stands. There was a significant negative correlation between SOC and mean annual temperature (MAT) and sand composition, in all types of forest stands. In contrast, the silt fraction has a positive correlation with SOC, in all types of tree stands. Variation in SOC under different types of forest stands in different landscapes can be due to differences in MAT, and the sand and silt fraction of soil apart from the type of forests.
\end{abstract}

Keywords: Coniferous, Deciduous, Evergreen, Sand, Silt

\section{Introduction}

Soil organic carbon (SOC) is an essential component of environmental quality assessment. Atmospheric $\mathrm{CO}_{2}$ is transferred into long-lasting pools, such as soil organic matter (SOM), thus reducing the atmospheric concentration of $\mathrm{CO}_{2}$ (McBratney et al. 2014). SOC is vital for soil fertility, plant growth, and production (Janzen 2006). The total amount of carbon $(C)$ stored in soil globally is estimated to be $1500 \mathrm{Pg} \mathrm{C}$, with soil containing more C than the atmosphere $(800 \mathrm{Pg} \mathrm{C})$ and vegetation $(500 \mathrm{Pg}$ C) combined (FAO \& ITPS 2015). A small variation in SOC concentrations can significantly affect the global carbon cycle (Walter et al. 2016).

Climate, land cover, soil texture, and soil order all affect SOC storage (Batjes 2016): Entisols and Aridisols store low amounts of SOC, whereas Histosols naturally store high amounts. When $\mathrm{CaCO}_{3}$ is present in the soil, typically at $\mathrm{pH} 6.5$ or higher, soil inorganic carbon (SIC) is formed (Lal et al. 1995). Histosols, Andisols, Spodosols, Oxisols, and Ultisols do not contain SIC, but Aridosols may store high amounts of SIC. On average,

Correspondence: mzut108@mzu.edu.in

Department of Environmental Science, Mizoram University, Aizawl, India
Inceptisols store the lowest amount of total C (SOC and SIC) and Histosols store the highest amount of total C (mostly SOC) (Eswaran et al. 2000). Global SOC concentrations range from low to high in soils of arid and temperate regions, respectively, and extremely high in organic or peat soils (Lal 2004).

Vegetation community structure may also affect the size of the SOC pool by altering both the microenvironment and soil characteristics (You et al. 2014). Globally, forest soil is a much more important $\mathrm{C}$ sink than live forest biomass, with concentrations two to four times higher in the upper $30 \mathrm{~cm}$, and three to six times higher in the upper $50 \mathrm{~cm}$ (Calvode et al. 2020). Worldwide, forests cover 4.03 billion ha, approximately $30 \%$ of the earth's surface: of the total C stock in forest biomes, 37\% occurs in low latitude forests, $14 \%$ in mid latitudes and $49 \%$ in high latitudes. A large part of the total SOC occurs in soils of tundra, pre-tundra, and taiga regions (Wyse 2012). Forests with different tree species vary in litter quality and root exudates, generating a divergence in soil properties, which may influence the soil microbial community (Chandra et al. 2016). SOC dynamics also differ due to variations in local vegetation types (Saiz et al. 2012; Gruba et al. 2015).

(c) The Author(s). 2021 Open Access This article is licensed under a Creative Commons Attribution 4.0 International License, which permits use, sharing, adaptation, distribution and reproduction in any medium or format, as long as you give appropriate credit to the original author(s) and the source, provide a link to the Creative Commons licence, and indicate if changes were made. The images or other third party material in this article are included in the article's Creative Commons licence, unless indicated otherwise in a credit line to the material. If material is not included in the article's Creative Commons licence and your intended use is not permitted by statutory regulation or exceeds the permitted use, you will need to obtain permission directly from the copyright holder. To view a copy of this licence, visit http://creativecommons.org/licenses/by/4.0/. 
Research into the effect of tree species on SOC is crucial for mitigating the effects of greenhouse gases (Jandl et al. 2007). Tree species are one of several factors that influence soil $\mathrm{C}$ and nitrogen $(\mathrm{N})$ inputs and outputs. Comparative studies of tree species grown under different conditions are beneficial in determining their effect (Binkley 1995), and the influence of tree species depends on the differences in soil conditions, such as parent material or land use (Vesterdal et al. 2008). Zhou et al. (2020) observed mixed forest stands of Cunninghamia lanceolata and Phyllostachys heterocycla have 3.33\% of SOM compared with pure stands of C. lanceolata (1.77\%) indicating mixed forest stands are better for storing SOM. Marler et al. (2016) have also indicated mixed deciduous tree stands can store more SOC $(130.0$ $\mathrm{mgg}^{-1}$ ) than pure forest stands of Leucaena leucophela $\left(73.7 \mathrm{mgg}^{-1}\right)$. Mixing of Acacia tree species in Eucalyptus plantations in sandy and nutrient-poor soils involved soil $\mathrm{C}$ and $\mathrm{N}$ accretion after 7 years in the Republic of Congo (Koutika et al. 2019). Similar observations of mixed forest stands recording more SOC than pure stands were also reported by Yao et al. (2019). While, deciduous forests, with large $\mathrm{C}$ pools in the forest floor, store less carbon in soil (Oostra et al. 2006), and more $C$ has been found in soils under mixed spruce forest in central western Europe (Berger et al. 2002). According to Guedes et al. (2016), coniferous tree stands of Pinus taeda recorded $135 \mathrm{Mg} / \mathrm{ha}$ of SOC compared with deciduous tree stands of Miombo (87 Mg/ha). Adivia et al. (2016) have provided consistent evidence of greater buildup of forest floor humus leading to more SOC in coniferous forest soils. Therefore, vegetation type is the most important variable driving the spatial pattern of SOC (Shi et al. 2012). Trees may influence the properties of soils beneath them, with a number of species affecting factors such as pH (Finzi et al. 1998), C and N levels, and the composition of the microbial community (Mitchell et al. 2010).

In this review, the author sought to determine whether variation in tree species is the only factor regulating the amount of SOC, or if other variables are involved. The effect of mean annual temperature (MAT), mean annual precipitation (MAP), tree age, elevation, soil $\mathrm{pH}$, and the relative composition of sand, silt, and clay were selected for analysis as these have been commonly reported in the literature. The aim of this review is to determine what effect these variables have on SOC.

\section{Methodology}

For this review, a literature survey was carried out using the following search engines and academic platforms: ResearchGate (https://www.researchgate.net), Google Scholar (www.googlescholar.com), Science Direct (https://www.sciencedirest.com), Springer (www. springer.com), and Taylor and Francis (https://www. taylorandfrancis.com). Research published prior to January 2020 was considered for the present study. The keywords used were "SOC in different tree stands" and "influence of trees on SOC." Numerous studies have reported SOC from different forest types; however, the specific tree species and the level of SOC in the forests are less reported. Therefore, literature on "litter decomposition," where soil characteristics have been reported, were also collected. In numerous studies, SOC has been given in the form of C-storage. This is difficult to convert into SOC without knowing the bulk density value and weight of soil particles $\geq 2 \mathrm{~mm}$, therefore these studies were not included.

The most widely reported procedure for SOC estimation is the wet digestion method of the Walkley-Black technique (Walkley 1947), which uses the heat from a sulfuric acid reaction to oxidize SOC by hot chromic acid. In recent studies, a CHN-elemental analyzer is used for SOC estimation. In many studies, a conversion factor of 1.2 has been applied for the SOC levels determined by the Walkley-Black method; in the present review, however, no conversion factor was applied as DeVos et al. (2007) have pointed out sandy soils with conifers showed 6\% higher recoveries than broadleaved species on heavier textured soils. For each laboratory and type of soil, they recommended that specific recovery factors need to be determined in order to standardize the results. Studies that reported only SOM were converted to SOC by dividing by 1.72 as SOM contains $58 \%$ SOC. In most of the studies, SOC levels were given in $\mathrm{g} \mathrm{kg}^{-1}$, which was converted into percentage by dividing by a factor of 10 .

The composition of sand, silt, and clay were determined either by the micro-pipette method (Miller and Miller 1987), the sieve method, or the hydrometer method (Day 1965). Studies that measured soil pH using a 1:1 or a 1:2.5 soil to water ratio were selected, as many studies have also reported the $\mathrm{pH}$ as the soil to $\mathrm{KCl}$ ratio. The soil sampling depths were variable (Adekunle et al. 2011; Guedes et al. 2016; Zhou et al.2020); however, the most common depth was $0-20 \mathrm{~cm}$, therefore, the present review was limited to reports from this depth. In all the selected studies, soil sampling was done by removal of the organic layer prior to sampling. As many as 60 tree species were recorded. Among the coniferous trees, the number of deciduous-conifer was very less compared with evergreen-conifers; therefore, they were grouped together as coniferous trees. The broadleaved-deciduous and broadleaved-evergreen trees were simply classified as deciduous and evergreen trees. Altogether the trees were broadly classified into four categories: coniferous, deciduous, evergreen, and mixed types. 
Table 1 Soil organic carbon (SOC) and associated variables (age, mean annual temperature [MAT], mean annual precipitation [MAP], soil type, altitude, depth, \% sand, \% silt, \% clay, and pH) in different types of tree stands, C: coniferous; D: deciduous; E: evergreen; and M: mixed. Results are shown in chronological order; ("do" indicates the same as above)

\begin{tabular}{|c|c|c|c|c|c|c|c|c|c|c|c|c|c|}
\hline $\begin{array}{l}\text { SI. } \\
\text { no. }\end{array}$ & Species name & $\begin{array}{l}\text { Age } \\
\text { (years) }\end{array}$ & $\begin{array}{l}\text { MAT } \\
\left({ }^{\circ} \mathrm{C}\right)\end{array}$ & $\begin{array}{l}\text { MAP } \\
(\mathrm{mm})\end{array}$ & Soil type & $\begin{array}{l}\text { Altitude } \\
\text { (m asl) }\end{array}$ & $\begin{array}{l}\text { Depth } \\
\text { (cm) }\end{array}$ & $\begin{array}{l}\text { Sand } \\
(\%)\end{array}$ & $\begin{array}{l}\text { Silt } \\
(\%)\end{array}$ & $\begin{array}{l}\text { Clay } \\
\text { (\%) }\end{array}$ & $\mathrm{pH}$ & $\begin{array}{l}\text { SOC } \\
(\%)\end{array}$ & Reference \\
\hline \multirow[t]{3}{*}{1.} & Acer saccharum (D) & 44 & & & Fragiocrept & & $0-10$ & & & & 4.00 & 5.9 & $\begin{array}{l}\text { Riha et al. } \\
\text { (1986) }\end{array}$ \\
\hline & Pinus resinosa $(\mathrm{C})$ & 44 & & & do & & & & & & 3.83 & 5.1 & do \\
\hline & Picea abies (C) & 44 & & & Dystrochrept & & & & & & 3.76 & 4.9 & do \\
\hline 2. & $\begin{array}{l}\text { Dipterocarpus tuberculatus } \\
\text { (D) }\end{array}$ & & 22.0 & 1245 & & $300-360$ & & 70.00 & 18.00 & 12.00 & 6.20 & 0.2 & $\begin{array}{l}\text { Yadava and } \\
\text { Devi (2007) }\end{array}$ \\
\hline \multirow[t]{3}{*}{3.} & Fagus sylvatica (D) & & & & & & $0-10$ & 82.00 & & & 3.70 & 5.0 & $\begin{array}{l}\text { Kooijman } \\
\text { et al. (2009) }\end{array}$ \\
\hline & do & & & & & & & 47.00 & & & 4.00 & 3.7 & do \\
\hline & do & & & & & & & 5.00 & & & 6.70 & 8.4 & do \\
\hline \multirow[t]{2}{*}{4.} & $\begin{array}{l}\text { Quercus leucotrichophora } \\
\text { (D) }\end{array}$ & & & & & 1566 & $0-20$ & & & & & 2.3 & $\begin{array}{l}\text { Sheikh et al. } \\
\text { (2009) }\end{array}$ \\
\hline & Pinus roxburghii (C) & & & & & 900 & do & & & & & 1.8 & \\
\hline 5. & $\begin{array}{l}\text { Fagus sylvatica and } \\
\text { Fraxinus excelsior (M) }\end{array}$ & 60 & 7.8 & $\begin{array}{l}750- \\
800\end{array}$ & Eutric cambisol & 440 & $0-10$ & 2.60 & 46.2 & 51.10 & & 5.3 & $\begin{array}{l}\text { Kutsch et al. } \\
\text { (2010) }\end{array}$ \\
\hline 6. & $\begin{array}{l}\text { Castanopsis carlesii and } \\
\text { Litsea acuminata (M) }\end{array}$ & & 18.2 & $\begin{array}{l}2342- \\
2743\end{array}$ & Inseptisol and Ultisol & $\begin{array}{l}670- \\
1000\end{array}$ & $0-20$ & 23.00 & 42.00 & 35.00 & 4.50 & 3.9 & $\begin{array}{l}\text { Owen et al. } \\
\text { (2010) }\end{array}$ \\
\hline \multirow[t]{4}{*}{7.} & Gmelina arborea (D) & 20 & & & & & $0-30$ & 34.71 & 29.76 & 20.53 & 7.30 & 2.7 & $\begin{array}{l}\text { Adekunle } \\
\text { et al. (2011) }\end{array}$ \\
\hline & do & 10 & & & & & & 21.04 & 21.76 & 27.20 & 7.33 & 1.9 & do \\
\hline & Tectona grandis (D) & 15 & & & & & & 68.73 & 19.23 & 12.04 & 8.33 & 1.1 & do \\
\hline & do & 8 & & & & & & 64.00 & 22.75 & 13.45 & 6.93 & 0.5 & \\
\hline 8. & $\begin{array}{l}\text { Shorea siamensis and Vitex } \\
\text { peduncularis }(M)\end{array}$ & & 25.0 & 1650 & Alfisol & $150-350$ & $0-13$ & 49.50 & 37.40 & 13.20 & 6.40 & 2.3 & $\begin{array}{l}\text { Takahashi } \\
\text { et al. (2011) }\end{array}$ \\
\hline 9. & Pinus densiflora (C) & 25 & 10.6 & 1245 & & 400 & $0-10$ & & & & $\begin{array}{l}5.2- \\
5.70\end{array}$ & 3.3 & $\begin{array}{l}\text { Yang et al. } \\
\text { (2011) }\end{array}$ \\
\hline \multirow[t]{2}{*}{10.} & Quercus mongolica (D) & & 4.6 & $\begin{array}{l}600- \\
1000\end{array}$ & Cryumbreps & 251 & $0-20$ & & & & 4.50 & 3.3 & $\begin{array}{l}\text { Sun et al. } \\
\text { (2012) }\end{array}$ \\
\hline & do & & 7.3 & do & Haplocryolls & 201 & do & & & & 5.6 & 4.4 & do \\
\hline \multirow[t]{5}{*}{11.} & $\begin{array}{l}\text { Cinnamomum } \\
\text { chekiangense (E) }\end{array}$ & 600 & 19.4 & 1731 & Red soil/hapludult & $390-430$ & $0-20$ & & & & 4.08 & 2.9 & $\begin{array}{l}\text { Yu et al. } \\
\text { (2012) }\end{array}$ \\
\hline & Castanopsis fargesii (E) & & & & & & & & & & 4.65 & 2.9 & do \\
\hline & Altingia gracilipes (E) & & & & & & & & & & 4.12 & 6.3 & do \\
\hline & $\begin{array}{l}\text { Tsoongiodendron odorum } \\
\text { (D) }\end{array}$ & & & & & & & & & & 4.00 & 3.33 & do \\
\hline & $\begin{array}{l}\text { Cunninghamia lanceolata } \\
\text { (C) }\end{array}$ & & & & & & & & & & 4.60 & 2.2 & do \\
\hline 12. & $\begin{array}{l}\text { Pseudotsuga menziesii, } \\
\text { Pinus lambertiana and } \\
\text { Pinus ponderosa (M) }\end{array}$ & 50 & & & & $\begin{array}{l}495- \\
1199\end{array}$ & $0-15$ & & & & & 8.3 & $\begin{array}{l}\text { Heckman } \\
\text { et al. (2013) }\end{array}$ \\
\hline \multirow[t]{4}{*}{13.} & Populous davidiana (D) & & 2.0 & 400 & & $\begin{array}{l}1250- \\
1300\end{array}$ & $0-10$ & & & & 6.14 & 10.6 & $\begin{array}{l}\text { Miao et al. } \\
\text { (2013) }\end{array}$ \\
\hline & $\begin{array}{l}\text { Populous davidiana and } \\
\text { Betula platyphylla (M) }\end{array}$ & & & & & $\begin{array}{l}1370- \\
1550\end{array}$ & & & & & 5.89 & 8.4 & do \\
\hline & Betula platyphylla (D) & & & & & $\begin{array}{l}1550- \\
1720\end{array}$ & & & & & 5.93 & 6.8 & do \\
\hline & Larix principis-rupprechtii (C) & & & & & $\begin{array}{l}1840- \\
1890\end{array}$ & & & & & 5.83 & 6.2 & do \\
\hline 14. & Calocedrus formosana (C) & $>40$ & 21.0 & $>$ & Dystrudept & 500 & $0-10$ & 5.10 & 45.30 & 49.70 & 3.80 & 6.1 & Lin et al. \\
\hline
\end{tabular}


Table 1 Soil organic carbon (SOC) and associated variables (age, mean annual temperature [MAT], mean annual precipitation [MAP], soil type, altitude, depth, \% sand, \% silt, \% clay, and pH) in different types of tree stands, C: coniferous; D: deciduous; E: evergreen; and M: mixed. Results are shown in chronological order; ("do" indicates the same as above) (Continued)

\begin{tabular}{|c|c|c|c|c|c|c|c|c|c|c|c|c|c|}
\hline $\begin{array}{l}\text { SI. } \\
\text { no. }\end{array}$ & Species name & $\begin{array}{l}\text { Age } \\
\text { (years) }\end{array}$ & $\begin{array}{l}\text { MAT } \\
\left({ }^{\circ} \mathrm{C}\right)\end{array}$ & $\begin{array}{l}\text { MAP } \\
(\mathrm{mm})\end{array}$ & Soil type & $\begin{array}{l}\text { Altitude } \\
\text { ( } \mathrm{m} \text { asl) }\end{array}$ & $\begin{array}{l}\text { Depth } \\
\text { (cm) }\end{array}$ & $\begin{array}{l}\text { Sand } \\
(\%)\end{array}$ & $\begin{array}{l}\text { Silt } \\
(\%)\end{array}$ & $\begin{array}{l}\text { Clay } \\
(\%)\end{array}$ & $\mathrm{pH}$ & $\begin{array}{l}\text { SOC } \\
(\%)\end{array}$ & Reference \\
\hline & & & & 4000 & & & & & & & & & (2014) \\
\hline & Cryptomeria japonica (C) & do & do & do & do & do & do & 6.6 & 45.00 & 48.40 & 3.90 & 5.3 & do \\
\hline 15. & $\begin{array}{l}\text { Pinus tabulaeformis and } \\
\text { Robinia pseudoacacia (M) }\end{array}$ & 20 & 10.8 & 602 & & $\begin{array}{l}900- \\
1300\end{array}$ & $0-20$ & $30-55$ & $\begin{array}{l}20- \\
45\end{array}$ & $\begin{array}{l}15- \\
25\end{array}$ & & 1.9 & $\begin{array}{l}\text { Liu et al. } \\
(2014)\end{array}$ \\
\hline \multirow[t]{3}{*}{16.} & Shorea robusta (D) & & 27.0 & 2021 & & 450 & $0-15$ & 66.01 & 14.12 & 19.87 & 6.21 & 1.8 & $\begin{array}{l}\text { Mandal and } \\
\text { Joshi (2014) }\end{array}$ \\
\hline & do & & & & & 600 & & 69.87 & 10.12 & 20.01 & 5.89 & 1.8 & do \\
\hline & do & & & & & 850 & & 55.44 & 26.11 & 18.45 & 5.69 & 1.6 & do \\
\hline 17. & $\begin{array}{l}\text { Albizia zygia and } \\
\text { Myrianthus arboreus (M) }\end{array}$ & & 23.5 & $\begin{array}{l}1350- \\
1550\end{array}$ & Oxisol & $650-700$ & $0-10$ & & & 58.30 & 4.60 & 1.9 & $\begin{array}{l}\text { Sugihara } \\
\text { et al. (2014) }\end{array}$ \\
\hline \multirow[t]{3}{*}{18.} & Larix kaempferi (C) & 17 & & & & & $0-20$ & & & & 5.39 & 4.2 & $\begin{array}{l}\text { Chen et al. } \\
\text { (2015) }\end{array}$ \\
\hline & do & 23 & & & & & & & & & 4.96 & 3.9 & do \\
\hline & Pinus armandii (C) & 25 & & & & & & & & & 5.35 & 4.4 & do \\
\hline 19. & Pinus pinaster (C) & 67 & 15.35 & 883 & Regosil & 6 & $0-10$ & 93.00 & 3.00 & 4.00 & 4.40 & 1.0 & $\begin{array}{l}\text { Matteucci } \\
\text { et al. (2015) }\end{array}$ \\
\hline \multirow[t]{3}{*}{20.} & Picea abies (C) & $\begin{array}{l}81- \\
100\end{array}$ & 7.4 & $\begin{array}{l}550- \\
700\end{array}$ & Leptic cambisols & $502-530$ & $0-10$ & 12.30 & 72.10 & 15.50 & 3.80 & 2.6 & $\begin{array}{l}\text { Andivia et al. } \\
\text { (2016) }\end{array}$ \\
\hline & Fagus sylvatica (D) & do & do & do & do & do & do & 20.8 & 65.6 & 13.6 & 4 & 2.8 & do \\
\hline & $\begin{array}{l}\text { Picea abies and Fagus } \\
\text { sylvatica (M) }\end{array}$ & do & do & do & do & do & do & 28.10 & 55.40 & 16.50 & 3.70 & 3.7 & do \\
\hline 21. & Quercus mongolica (D) & & 10.8 & 1170 & & 1424 & $0-20$ & 27.80 & 19.40 & 52.80 & 4.60 & 6.9 & $\begin{array}{l}\text { Chae et al. } \\
(2016)\end{array}$ \\
\hline \multirow[t]{5}{*}{22.} & Shorea robusta (D) & & 26.0 & 73.1 & & 265 & $0-10$ & 66.70 & 6.00 & 27.33 & 6.36 & 0.4 & $\begin{array}{l}\text { Chandra } \\
\text { et al. (2016) }\end{array}$ \\
\hline & Sal mixed & & 26.0 & do & & 258 & & 60.00 & 20.67 & 19.33 & 6.33 & 0.9 & do \\
\hline & Pinus roxburghii (C) & & 16.5 & 151.9 & & 1822 & & 73.33 & 9.33 & 17.33 & 6.48 & 4.5 & do \\
\hline & Quercus leucotrichophor (D) & & 16.5 & do & & 1333 & & 48.67 & 16.67 & 34.67 & 6.88 & 2.8 & do \\
\hline & Oak mixed & & 16.5 & do & & 1305 & & 52.67 & 8.00 & 39.33 & 6.61 & 4.2 & do \\
\hline \multirow[t]{3}{*}{23.} & Brachystegia boehmii (E) & 34 & 21.2 & 1300 & $\begin{array}{l}\text { Ferrasol and } \\
\text { cambisol }\end{array}$ & & $0-10$ & & & & $\begin{array}{l}5.1- \\
5.3\end{array}$ & 1.8 & $\begin{array}{l}\text { Guedes et al. } \\
\text { (2016) }\end{array}$ \\
\hline & Pinus taeda $(\mathrm{C})$ & do & do & do & do & & do & & & & $\begin{array}{l}5.1- \\
5.3\end{array}$ & 3.8 & do \\
\hline & Eucalyptus grandis (E) & do & do & do & do & & do & & & & $\begin{array}{l}5.9- \\
6.0\end{array}$ & 3.7 & do \\
\hline 24. & Leucaena leucocephala (E) & & & & & & & & & & 7.15 & 7.4 & $\begin{array}{l}\text { Marler et al. } \\
(2016)\end{array}$ \\
\hline \multirow[t]{8}{*}{25.} & Picea smithiana (C) & & $<1.0$ & $\begin{array}{l}254- \\
400\end{array}$ & & 2860 & & & & & & 3.3 & $\begin{array}{l}\text { Shedayi } \\
\text { et al. (2016) }\end{array}$ \\
\hline & do & & do & do & & 3260 & & & & & & 6.8 & do \\
\hline & Pinus wallichiana $(\mathrm{C})$ & & do & do & & 2890 & & & & & & 2.2 & do \\
\hline & do & & do & do & & 3350 & & & & & & 2.4 & do \\
\hline & Juniperus excelsa (C) & & do & do & & 3080 & & & & & & 1.7 & do \\
\hline & do & & do & do & & 3170 & & & & & & 0.7 & do \\
\hline & Betula utilis (D) & & do & do & & 3290 & & & & & & 2.1 & do \\
\hline & & & do & do & & 3440 & & & & & & 7.5 & \\
\hline 26. & Abies alba (C) & $70-90$ & & & Dystric cambisol & $388-407$ & $0-10$ & 32.8 & 60.7 & 6.49 & 4.28 & 4.6 & Ewa Blonska \\
\hline
\end{tabular}


Table 1 Soil organic carbon (SOC) and associated variables (age, mean annual temperature [MAT], mean annual precipitation [MAP], soil type, altitude, depth, \% sand, \% silt, \% clay, and pH) in different types of tree stands, C: coniferous; D: deciduous; E: evergreen; and M: mixed. Results are shown in chronological order; ("do" indicates the same as above) (Continued)

\begin{tabular}{|c|c|c|c|c|c|c|c|c|c|c|c|c|c|}
\hline $\begin{array}{l}\text { Sl. } \\
\text { no. }\end{array}$ & Species name & $\begin{array}{l}\text { Age } \\
\text { (years) }\end{array}$ & $\begin{array}{l}\text { MAT } \\
\left({ }^{\circ} \mathrm{C}\right)\end{array}$ & $\begin{array}{l}\text { MAP } \\
(\mathrm{mm})\end{array}$ & Soil type & $\begin{array}{l}\text { Altitude } \\
\text { (m asl) }\end{array}$ & $\begin{array}{l}\text { Depth } \\
\text { (cm) }\end{array}$ & $\begin{array}{l}\text { Sand } \\
(\%)\end{array}$ & $\begin{array}{l}\text { Silt } \\
(\%)\end{array}$ & $\begin{array}{l}\text { Clay } \\
(\%)\end{array}$ & $\mathrm{pH}$ & $\begin{array}{l}\text { SOC } \\
(\%)\end{array}$ & Reference \\
\hline & & & & & & & & & & & & & et al. (2017) \\
\hline & Fagus sylvatica (D) & do & & & do & do & do & 38.98 & 55.23 & 5.29 & 4.56 & 3.0 & do \\
\hline \multirow[t]{2}{*}{27.} & Acacia confusa (E) & & 23.0 & 1347 & & 110 & $0-10$ & & & & $\begin{array}{l}3.8- \\
3.9\end{array}$ & $\begin{array}{l}4.5- \\
4.9\end{array}$ & $\begin{array}{l}\text { Huang et al. } \\
\text { (2017) }\end{array}$ \\
\hline & Cryptomeria japonica (C) & 60 & 16.6 & 2635 & & 1250 & do & & & & 5.80 & 3.4 & do \\
\hline \multirow[t]{2}{*}{28.} & $\begin{array}{l}\text { Pinus massoniana and } \\
\text { Lithocarpus glaber (M) }\end{array}$ & & 17.3 & 1416 & Acrisol & $55-260$ & $0-10$ & & & & & 1.6 & $\begin{array}{l}\text { Ouyang } \\
\text { et al. (2017) }\end{array}$ \\
\hline & $\begin{array}{l}\text { Lithocarpus glaber and } \\
\text { Cyclobalanopsis glauca (M) }\end{array}$ & & 17.3 & 1416 & Acrisol & $55-260$ & $0-10$ & & & & & 2.4 & do \\
\hline 29. & Pinus pinaster (C) & & & & & & $0-15$ & 94.7 & 2.6 & 2.7 & $\begin{array}{l}4.1- \\
4.8\end{array}$ & 3.1 & $\begin{array}{l}\text { Suaze et al. } \\
(2017)\end{array}$ \\
\hline \multirow[t]{2}{*}{30.} & Albizia zygia (D) & $20-30$ & 23.5 & $\begin{array}{l}1350- \\
1550\end{array}$ & Oxisol & $650-700$ & $0-10$ & & & 54.90 & 4.80 & 2.5 & $\begin{array}{l}\text { Sugihara } \\
\text { et al. (2017) }\end{array}$ \\
\hline & do & 50 & do & do & do & do & do & & & 60.60 & 4.70 & 1.9 & do \\
\hline 31. & Araucaria angustifolia (C) & & 17.0 & $\begin{array}{l}1600- \\
1800\end{array}$ & Humic Cambisol & 820 & $0-5$ & 21.00 & 29.00 & 50.00 & 3.80 & 4.6 & $\begin{array}{l}\text { Thomaz } \\
\text { (2017) }\end{array}$ \\
\hline \multirow[t]{4}{*}{32.} & Fagus sylvatica (D) & & & 800 & Sandy & & & & & & 3.40 & 5.6 & $\begin{array}{l}\text { Weemstra } \\
\text { et al. (2017) }\end{array}$ \\
\hline & do & & & & Clayey & & & & & & 6.80 & 3.8 & do \\
\hline & Picea abies (C) & & & & Sandy & & & & & & 3.40 & 6.0 & do \\
\hline & do & & & & Clayey & & & & & & 6.60 & 4.8 & do \\
\hline 33. & $\begin{array}{l}\text { Picea abies and Larix } \\
\text { decidua (M) }\end{array}$ & 110 & 7.8 & 1645 & $\begin{array}{l}\text { Lithic to rendzic } \\
\text { leptosols and } \\
\text { chromic cambisols }\end{array}$ & 950 & $0-10$ & & & & & 9.4 & $\begin{array}{l}\text { Zehetgruber } \\
\text { et al. (2017) }\end{array}$ \\
\hline 34. & Pinus jeffreyi (C) & & & $\begin{array}{l}1800- \\
3500\end{array}$ & & & $5-8$ & 74.28 & 18.04 & 7.65 & 5.41 & 1.8 & $\begin{array}{l}\text { Fry et al. } \\
\text { (2018) }\end{array}$ \\
\hline \multirow[t]{2}{*}{35.} & $\begin{array}{l}\text { Picea abies and Pinus } \\
\text { cembra (M) }\end{array}$ & & 11.0 & 1000 & Haplic podsol & 1737 & & & & & 3.30 & 11.0 & $\begin{array}{l}\text { Margesin } \\
\text { et al. (2016) }\end{array}$ \\
\hline & $\begin{array}{l}\text { Pinus sylvestris and Quercus } \\
\text { pubenscens (M) }\end{array}$ & & do & 900 & Drystic cambisol & 570 & & & & & 4.10 & 4.1 & do \\
\hline \multirow[t]{2}{*}{36.} & Castanopsis carlesii (E) & & 19.0 & 1749 & Ferralic cambisols & & $0-10$ & 60.5 & 13.82 & 25.69 & 4.33 & 2.8 & $\begin{array}{l}\text { Si et al. } \\
\text { (2018) }\end{array}$ \\
\hline & Castanopsis fargesii (E) & & do & 1688 & Humic alfisol & & do & 37.29 & 43.55 & 19.17 & 4.64 & 6.8 & \\
\hline \multirow[t]{2}{*}{37.} & Albizia zygia (D) & & 23.5 & $\begin{array}{l}1350- \\
1550\end{array}$ & & $600-650$ & $0-10$ & & & 55.20 & 4.40 & 1.8 & $\begin{array}{l}\text { Sugihara } \\
\text { et al. (2018) }\end{array}$ \\
\hline & do & & & & & & & & & & & & do \\
\hline \multirow[t]{2}{*}{38.} & Podocarpus falcatus (C) & & 21.0 & $\begin{array}{l}1700- \\
2824\end{array}$ & Oxisols & $\begin{array}{l}900- \\
1500\end{array}$ & $0-15$ & 34.33 & 49 & 16.42 & 5.82 & 4.7 & $\begin{array}{l}\text { Tellen and } \\
\text { Yerima } \\
\text { (2018) }\end{array}$ \\
\hline & $\begin{array}{l}\text { Eucalyptus salinga and } \\
\text { Eucalyptus grandis (M) }\end{array}$ & & do & do & do & do & do & 36 & 49.2 & 14.5 & 5.61 & 4.3 & do \\
\hline \multirow[t]{3}{*}{39.} & Quercus ilex (D) & & 17 & & & $\begin{array}{l}1600- \\
2200\end{array}$ & & & & & & 3.2 & $\begin{array}{l}\text { Ali et al. } \\
\text { (2019) }\end{array}$ \\
\hline & $\begin{array}{l}\text { Pinus wallichiana and } \\
\text { Cedrus deodara (M) }\end{array}$ & & & & & $\begin{array}{l}1600- \\
3100\end{array}$ & & & & & & 4.1 & do \\
\hline & Pinus roxbhurghii (C) & & & & & $\begin{array}{l}800- \\
1600\end{array}$ & & & & & & 2.4 & do \\
\hline 40. & Acacia mangium (E) & 7 & 25.0 & 1200 & Ferralic arenosol & 100 & $0-5$ & $>90$ & 2.00 & 6.00 & 4.20 & 2.2 & $\begin{array}{l}\text { Koutika et al. } \\
\text { (2019) }\end{array}$ \\
\hline
\end{tabular}


Table 1 Soil organic carbon (SOC) and associated variables (age, mean annual temperature [MAT, mean annual precipitation [MAP], soil type, altitude, depth, \% sand, \% silt, \% clay, and pH) in different types of tree stands, C: coniferous; D: deciduous; E: evergreen; and M: mixed. Results are shown in chronological order; "do" indicates the same as above) (Continued)

\begin{tabular}{|c|c|c|c|c|c|c|c|c|c|c|c|c|c|}
\hline $\begin{array}{l}\text { Sl. } \\
\text { no. }\end{array}$ & Species name & $\begin{array}{l}\text { Age } \\
\text { (years) }\end{array}$ & $\begin{array}{l}\text { MAT } \\
\left({ }^{\circ} \mathrm{C}\right)\end{array}$ & $\begin{array}{l}\text { MAP } \\
(\mathrm{mm})\end{array}$ & Soil type & $\begin{array}{l}\text { Altitude } \\
\text { (m asl) }\end{array}$ & $\begin{array}{l}\text { Depth } \\
\text { (cm) }\end{array}$ & $\begin{array}{l}\text { Sand } \\
(\%)\end{array}$ & $\begin{array}{l}\text { Silt } \\
(\%)\end{array}$ & $\begin{array}{l}\text { Clay } \\
\text { (\%) }\end{array}$ & $\mathrm{pH}$ & $\begin{array}{l}\text { SOC } \\
(\%)\end{array}$ & Reference \\
\hline & $\begin{array}{l}\text { Eucalyptus urophyla and } \\
\text { Eucalyptus grandis (M) }\end{array}$ & do & 25.0 & do & & & & & & & & 2.2 & do \\
\hline & $\begin{array}{l}\text { Acacia and Eucalyptus 50: } \\
50(\mathrm{M})\end{array}$ & & & & do & & & & & & & 2.3 & do \\
\hline 41. & Pinus densiflora (C) & & 25.0 & 1437 & & 170 & $0-10$ & & & & & 4.3 & $\begin{array}{l}\text { Lee et al. } \\
\text { (2019) }\end{array}$ \\
\hline \multirow[t]{3}{*}{42.} & Pinus koraiens (C) & & $<1$ & $\begin{array}{l}800- \\
1800\end{array}$ & Dark brown & 699 & $0-20$ & 23.25 & 45.10 & 31.60 & 6.12 & 5.9 & $\begin{array}{l}\text { Liu et al. } \\
\text { (2019) }\end{array}$ \\
\hline & do & & & & & 937 & & 18 & 47.2 & 34.7 & 6.00 & 5.4 & do \\
\hline & do & & & & & 1177 & & 16.3 & 40.2 & 43.4 & 5.50 & 3.7 & do \\
\hline 43. & $\begin{array}{l}\text { Fagus orientalis and } \\
\text { Carpinus betulus (M) }\end{array}$ & & 15.4 & $\begin{array}{l}528- \\
817\end{array}$ & $\begin{array}{l}\text { Umbric fluvisol } \\
\text { Chromic cambisol }\end{array}$ & 210-995 & $0-9$ & 10.5 & 57 & 32 & & 4.0 & $\begin{array}{l}\text { Moslehi } \\
\text { et al. (2019) }\end{array}$ \\
\hline \multirow[t]{2}{*}{44.} & $\begin{array}{l}\text { Machilus calcicola and } \\
\text { Styrax suberifolius (M) }\end{array}$ & & 12.7 & 1067 & & & $0-10$ & & & & 6.58 & 5.3 & $\begin{array}{l}\text { Sheng et al. } \\
\text { (2019) }\end{array}$ \\
\hline & $\begin{array}{l}\text { Quercus aliena and } \\
\text { Carpinus viminea (M) }\end{array}$ & & 9.5 & 1234 & & & do & & & & 5.35 & 2.8 & do \\
\hline \multirow[t]{5}{*}{45.} & $\begin{array}{l}\text { Castanopsis chinensis and } \\
\text { Schima superba (M) }\end{array}$ & 400 & 20.7 & 1996 & Ferralic cambisol & $250-350$ & $0-20$ & 27.60 & 58.60 & 13.70 & 4.20 & 5.6 & $\begin{array}{l}\text { Sun et al. } \\
\text { (2019) }\end{array}$ \\
\hline & Pinus massoniana (C) & 60 & 20.9 & 1990 & Ferralic cambisol & $50-150$ & $0-20$ & 44.70 & 46.80 & 8.40 & 4.00 & 5.5 & do \\
\hline & Quercus aliena (D) & & 15.1 & 855 & Drystic cambisol & $\begin{array}{l}1380- \\
1400\end{array}$ & $0-20$ & 24.00 & 62.90 & 3.50 & 4.60 & 5.9 & do \\
\hline & $\begin{array}{l}\text { Quercus aliena and Pinus } \\
\text { armandi (M) }\end{array}$ & 64 & 15.1 & 855 & do & $\begin{array}{l}1330- \\
1360\end{array}$ & $0-20$ & 6.00 & 83.10 & 10.90 & 4.60 & 7.0 & do \\
\hline & $\begin{array}{l}\text { Betula platyphylla and } \\
\text { Populous davidiana (M) }\end{array}$ & & 3.6 & 689 & Eutric cambisol & 729-781 & & 7.40 & 86.20 & 6.40 & 5.50 & 9.0 & do \\
\hline \multirow[t]{5}{*}{46.} & Acacia mangium (E) & 6 & 27.0 & 950 & Grayish agrisol & & $0-10$ & 92.44 & 1.87 & 5.69 & 5.40 & 1.0 & $\begin{array}{l}\text { Weber et al. } \\
(2019)\end{array}$ \\
\hline & $\begin{array}{l}\text { Anadenanthera colubrina } \\
\text { (E) }\end{array}$ & do & do & do & do & do & do & do & do & do & do & 1.2 & do \\
\hline & Casuarina equisetifolia (E) & & & & & & & & & & & 1.2 & do \\
\hline & Eucalyptus urophylla (E) & & & & & & & & & & & 0.88 & do \\
\hline & Astronium fraxinifolium (D) & & & & & & & & & & & 0.8 & do \\
\hline \multirow[t]{2}{*}{47.} & $\begin{array}{l}\text { Michelia champaca and } \\
\text { Castanopsis sp. (M) }\end{array}$ & & & & & $\begin{array}{l}2300- \\
3000\end{array}$ & $0-10$ & 71.40 & 22.82 & 5.78 & 4.88 & 5.0 & $\begin{array}{l}\text { Yam et al. } \\
\text { (2019) }\end{array}$ \\
\hline & Cryptomeria japonica (C) & 60 & 16.6 & 2635 & & 1250 & do & & & & 5.80 & 3.4 & do \\
\hline 48. & Pinus massoniana (C) & & 19.0 & 1621 & Red soil & & $0-20$ & & & & & 1.9 & $\begin{array}{l}\text { Yao et al. } \\
\text { (2019) }\end{array}$ \\
\hline 49. & Pinus massoniana (C) & 11 & 18.1 & 1022 & Yellow & 453 & $0-10$ & & & & 3.98 & 1.6 & $\begin{array}{l}\text { Zhang et al. } \\
\text { (2019) }\end{array}$ \\
\hline 50. & Oak field (D) & & & $\begin{array}{l}1300- \\
1500\end{array}$ & Alluvial sediment & & $0-10$ & 10.00 & 77.00 & 13.00 & 5.60 & 1.9 & $\begin{array}{l}\text { Zhou et al. } \\
\text { (2019) }\end{array}$ \\
\hline \multirow[t]{2}{*}{51.} & $\begin{array}{l}\text { Ficus ovate and Sapium } \\
\text { ellipticum (M) }\end{array}$ & & 20.0 & $\begin{array}{l}1272- \\
1397\end{array}$ & Vertisol and lithosol & & $0-15$ & & & & 6.54 & 3.8 & $\begin{array}{l}\text { Kasa et al. } \\
(2019)\end{array}$ \\
\hline & Eucalyptus sp. (E) & & do & do & do & & do & & & & 6.31 & 2.1 & do \\
\hline 52. & Prosopsis juliflora (E) & & & & & & $0-10$ & & & & & 3.9 & $\begin{array}{l}\text { Meena et al. } \\
(2020)\end{array}$ \\
\hline \multirow[t]{2}{*}{53.} & $\begin{array}{l}\text { Tectona grandis SOC } \\
\text { Within } 1.5 \mathrm{~m}(\mathrm{D})\end{array}$ & 9 & & & & & $0-10$ & & & & & 0.8 & $\begin{array}{l}\text { Ikhajeagbe } \\
\text { et al. (2020) }\end{array}$ \\
\hline & Tectona grandis SOC & & & & & & & & & & & 0.4 & do \\
\hline
\end{tabular}


Table 1 Soil organic carbon (SOC) and associated variables (age, mean annual temperature [MAT, mean annual precipitation [MAP], soil type, altitude, depth, \% sand, \% silt, \% clay, and pH) in different types of tree stands, C: coniferous; D: deciduous; E: evergreen; and M: mixed. Results are shown in chronological order; ("do" indicates the same as above) (Continued)

\begin{tabular}{|c|c|c|c|c|c|c|c|c|c|c|c|c|c|}
\hline $\begin{array}{l}\text { Sl. } \\
\text { no. }\end{array}$ & Species name & $\begin{array}{l}\begin{array}{l}\text { Age } \\
\text { (years) }\end{array} \\
\end{array}$ & $\begin{array}{l}\text { MAT } \\
\left({ }^{\circ} \mathrm{C}\right)\end{array}$ & $\begin{array}{l}\text { MAP } \\
(\mathrm{mm})\end{array}$ & Soil type & $\begin{array}{l}\text { Altitude } \\
\text { (m asl) }\end{array}$ & $\begin{array}{l}\text { Depth } \\
(\mathrm{cm})\end{array}$ & $\begin{array}{l}\text { Sand } \\
(\%)\end{array}$ & $\begin{array}{l}\text { Silt } \\
(\%)\end{array}$ & $\begin{array}{l}\text { Clay } \\
(\%)\end{array}$ & $\mathrm{pH}$ & $\begin{array}{l}\text { SOC } \\
\text { (\%) }\end{array}$ & Reference \\
\hline & outside $1.5 \mathrm{~m}$ & & & & & & & & & & & & \\
\hline 54. & Casuarina equisetifolia (E) & $6-8$ & 25.0 & 1300 & & $6-8$ & $0-5$ & Sandy & & & 7.10 & 0.3 & Panda (2020) \\
\hline 55. & Quercus rubra (D) & & 8.0 & $\begin{array}{l}700- \\
800\end{array}$ & & & $0-15$ & & & & 3.70 & 1.1 & $\begin{array}{l}\text { Stanek et al. } \\
(2020)\end{array}$ \\
\hline
\end{tabular}

Data were summarized by the mean, maximum, minimum, and standard deviation. The Pearson's correlation coefficient was used to find associations between SOC and the following variables: MAT; MAP; sand, silt, and clay composition; age; elevation, and soil $\mathrm{pH}$. A Student's $t$ test was performed to determine the significance level $(p<0.05)$ for these correlations. A one-way analysis of variance was performed on the SOC levels in the different categories of trees using SPSS (IBM, version 16.0).

\section{Results and discussion}

\section{SOC under different tree stands}

SOC was found in very high concentrations under specific tree stands. Table 1 shows that the maximum level of SOC (11.02\%) was found in mixed stands of the coniferous trees Picea abies and Pinus cembra (Margesin et al. 2016), followed by mixed coniferous stands of Picea abies and Larix decidua (9.44\%) (Zehetgruber et al. 2017). Mixed deciduous stands of Betula platyphylla and Populus davidiana (Sun et al. 2019), deciduous stands of Fagus sylvatica (Kooijman et al. 2009), mixed coniferous stands of Pseudotsuga menzeisii, Pinus lambertiana and Pinus ponderosa (Heckman et al. 2013), deciduous stands of Betula utilis (Shedayi et al. 2016), and evergreen stands of Leuceana leucocephala (Marler et al. 2016) had SOC concentrations of 9.03\%, 8.4\%, 8.3\%, 7.57\%, and $7.37 \%$, respectively. The lowest concentration among the higher group was found in coniferous and deciduous mixed stands of Quercus aliena and Pinus armandi (7.01\%) (Sun et al. 2019).

Table 2 Mean and standard deviation of soil organic carbon (SOC) for all recorded tree species and different categories of tree stands ( $p<0.058$, one-way ANOVA)

\begin{tabular}{llllll}
\hline SOC(\%) & Max & Min & Mean & SD & $\boldsymbol{N}$ \\
\hline All tree species & 11.02 & 0.2 & 3.70 & 1.80 & 119 \\
Coniferous & 6.16 & 0.32 & 3.63 & 1.49 & 34 \\
Deciduous & 8.4 & 0.2 & 3.14 & 1.7 & 35 \\
Evergreen & 7.37 & 0.88 & 3.28 & 1.66 & 14 \\
Mixed & 11.02 & 0.9 & 4.62 & 2.08 & 27 \\
\hline
\end{tabular}

The results show that, out of the top eight tree stands that recorded the highest SOC level, three were from mixed coniferous tree stands: one from mixed deciduous trees, one from mixed coniferous and deciduous tree stands. The mixed stands recorded an average SOC of $4.62 \% \pm 2.08 \%$, with a range of $0.9 \%$ to $11.02 \%(p<0.058)$. The average SOC of mixed tree stands was higher than the overall average of all trees $(3.70 \%)$ (Table 2). These results indicate that mixed tree stands are common and can store the most SOC.

Within the coniferous tree stands, an average SOC of $3.63 \% \pm 1.49 \%$ was recorded: Larix principallis from Northeast China had the maximum (6.16\%) (Miao et al. 2013) and Juneperus excelsa from Northern Pakistan, had the least (0.70\%) (Shedayi et al. 2016). In deciduous tree stands, which had an average SOC of $3.14 \% \pm 1.70 \%$, Fagus sylvatica had the maximum (8.4\%) (Kooijman et al. 2009) followed by Betula utilis (7.57\%) (Shedayi et al. 2016), Quercus mongolica (6.9\%) (Chae et al. 2016), Betula platyphylla (6.8\%) (Miao et al. 2013) and Dipterocarpus tuberculatus had the least $(0.2 \%)$ (Yadava and Devi 2007). In evergreen tree stands, the maximum SOC level was 7.37\% in Leuceana leucocephalla (Marler et al. 2016), followed by $6.8 \%$ in Castanopsis fargesii ( $\mathrm{Si}$ et al. 2018), and the minimum level was $0.32 \%$ in Casuarina equisetifolia (Panda 2020); the average was $3.28 \% \pm$ $1.66 \%$.

The analysis of variance showed variation of SOC among the four types of stands $\left(F_{3,106}=2.56 ; P<\right.$ 0.058). SOC levels were most commonly reported from deciduous tree stands, with 35 concentrations recorded from different locations, followed by 34 recordings from coniferous tree stands. SOC levels from mixed tree stands were reported from 27 different locations, and only 14 evergreen tree stands had SOC levels recorded. As shown in Fig. 1, mixed tree stands can store the highest concentrations of SOC. Coniferous tree stands recorded higher amounts of SOC than deciduous trees, while evergreen tree stands recorded the lowest SOC level; however, this could also be due to fewer observations.

Coniferous species generally accumulate greater SOC concentrations in the forest floor layer than deciduous 


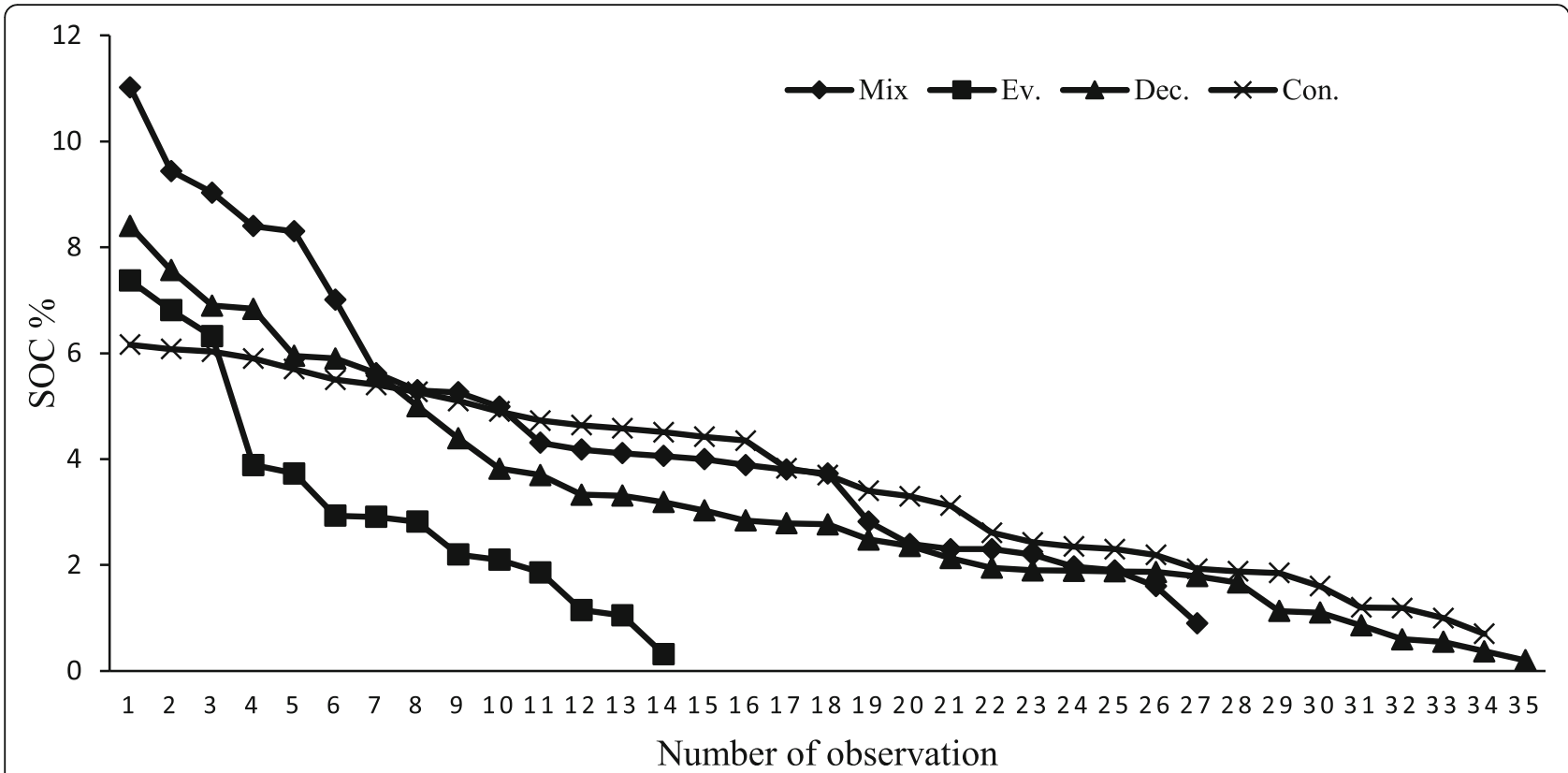

Fig. 1 Soil organic carbon (SOC) content in the four categories of tree stands. Mix: mixed tree; Ev: evergreen; Dec: deciduous; Con: coniferous

species (Augusto et al. 2015). Soil C reserves in the forest floor are generally greater under conifers than under broadleaved species (Vesterdal et al. 2013). As the needles of conifers take more time to decompose subsequent buildup of the litter can lead to more SOC, moreover conifers commonly occur in colder regions contributing to the delayed rate of decomposition. Disturbance due to human activities leading to erosion of soil is also an important factor in lowering SOC, which can be relatively lower in cold regions compared with warmer regions. Another factor can be attributed to the low amount of precipitation passing through a dense and low canopy in coniferous forests that prevented nutrient loss from the soil organic horizons (Lukina et al. 2019). Combining different tree species can have a profound effect on $\mathrm{C}$ accumulation ratios and $\mathrm{SOC}$ distribution within the soil profile (Chapin 2003), and compared with monospecific stands, the establishment of mixed forests promotes soil $\mathrm{C}$ sequestration. In mixed Norway spruce and European beech tree stands, the Norway spruce favored SOC accumulation in the forest floor whereas $\mathrm{C}$ incorporation into the uppermost mineral soil was promoted by root turnover of European beech. Therefore, conversion of monospecific plantations into mixed stands enhance SOM accumulation and stabilization in the mineral layers and, hence, the long-

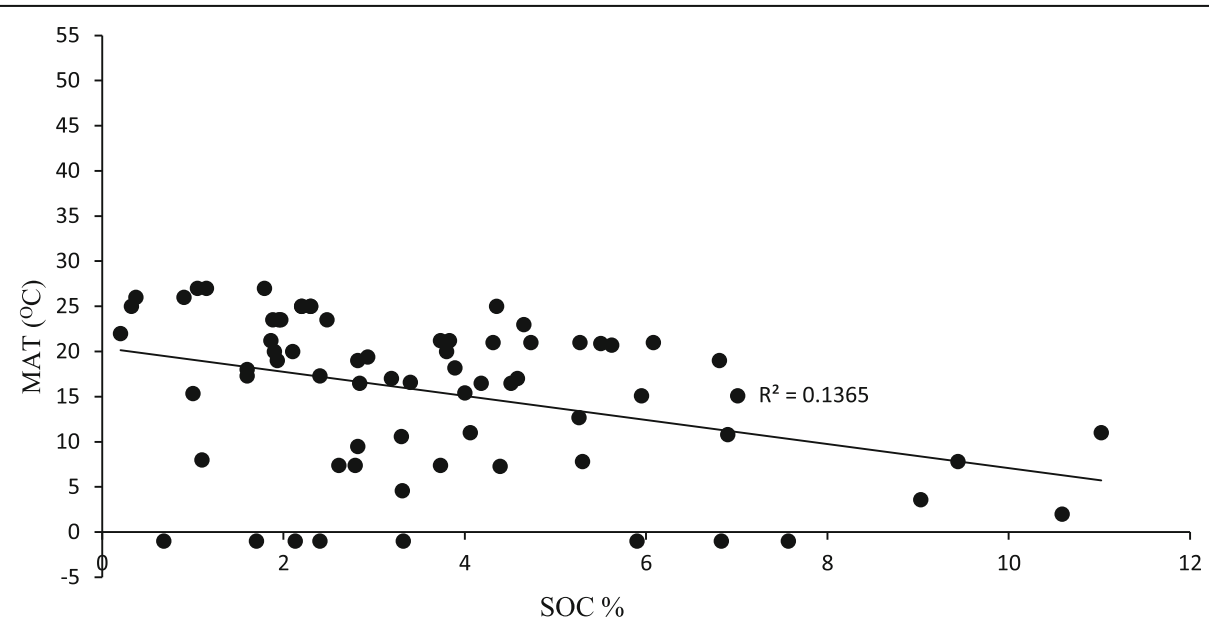

Fig. 2 Relationship of soil organic carbon (SOC) with mean annual temperature (MAT) (N=71) 


\section{- Age}

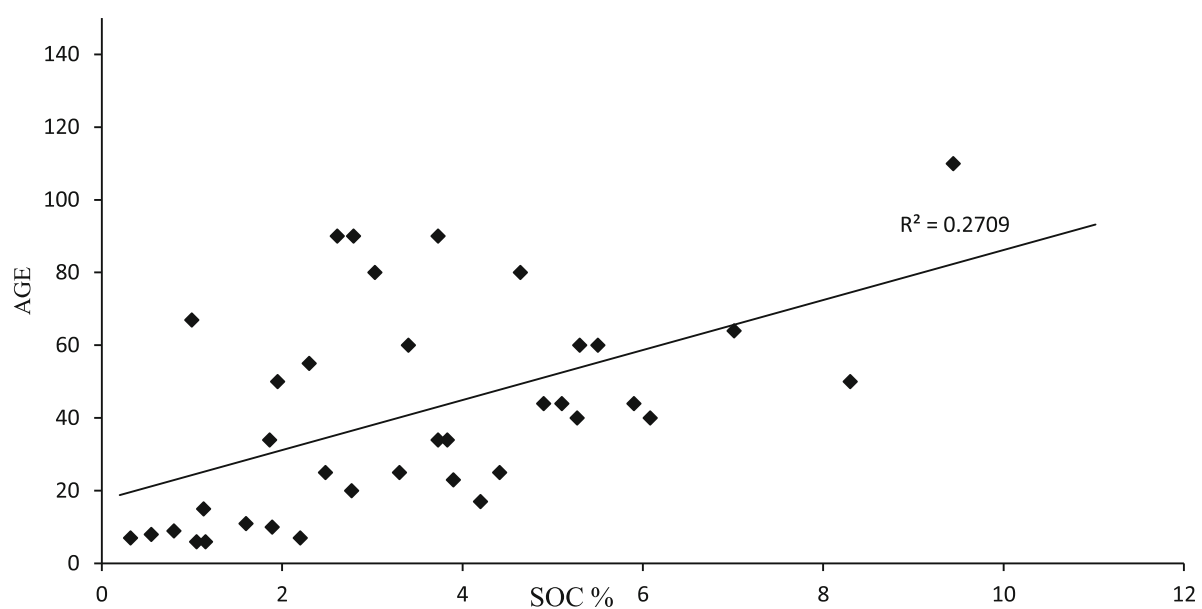

Fig. 3 Relationship of soil organic carbon $(\mathrm{SOC})$ with age $(N=38)$ for all the recorded tree species

term storage of $C$ (Andivia et al. 2016). The mixed type of tree stands provides a different composition in the input litter, regulating growth and survival of different types of soil macro- and microorganisms.

\section{Soil type}

The main soil type was found to be Cambisol, along with different subcategories, followed by Oxisol. Cambisols have a number of important characteristics that enable them to occur in widely differing environments: they contain weatherable minerals in the silt and sand fractions, have good water holding capacity, and have a neutral to weakly acidic soil reaction that promotes chemical fertility and an active soil fauna (Driessen
2001). Oxisols occur in the hot and humid conditions of tropical regions, where the $\mathrm{B}$ horizon is enriched with iron, aluminum oxides, and kaolinite (Beinroth et al. 1996).

\section{Role of MAT and MAP}

For all the tree types, SOC was significantly correlated with MAT $(p<0.05) \quad$ (Table 3), with a lower air temperature resulting in a higher SOC content (Fig. 2). This correlation was also significant in deciduous and mixed tree stands. Sun et al. (2019) observed an average rate of reduction in SOC was $1.87 \%$ with $1{ }^{\circ} \mathrm{C}$ increase in MAT in monospecific stands and mixed tree stands. However, annual litter fall (input) and soil microbial

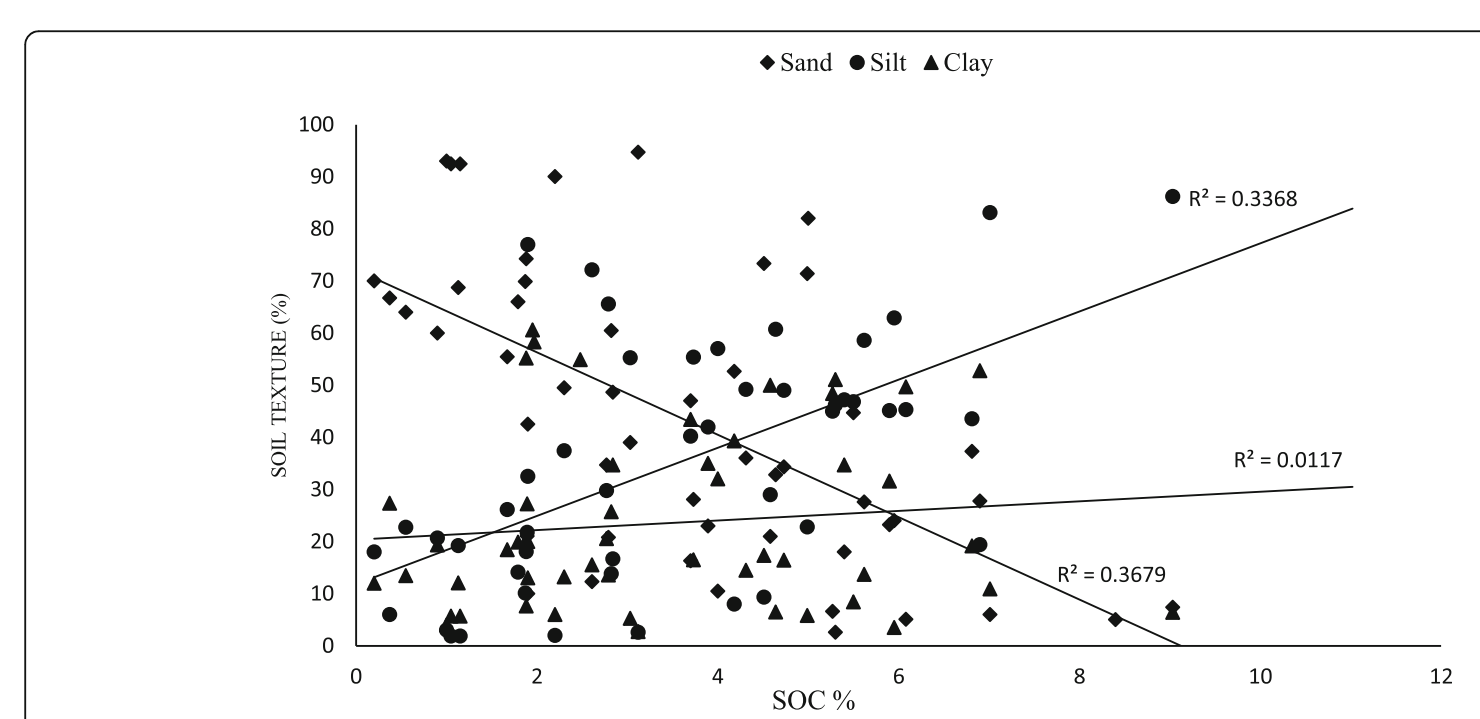

Fig. 4 Relationship of soil organic carbon (SOC) with sand $(N=50)$, silt $(N=47)$, and clay $(N=51)$ for all the recorded tree species 
Table 3 Correlation coefficient between soil organic carbon (SOC) and mean annual precipitation (MAP), mean annual temperature (MAT), sand, silt, clay, age, altitude, and pH for all tree species and the four categories of tree stands

\begin{tabular}{|c|c|c|c|c|c|c|c|c|c|c|}
\hline & \multicolumn{2}{|c|}{ All tree species } & \multicolumn{2}{|c|}{ Coniferous } & \multicolumn{2}{|c|}{ Deciduous } & \multicolumn{2}{|c|}{ Evergreen } & \multicolumn{2}{|l|}{ Mixed } \\
\hline & $\overline{R^{2}(\%)}$ & $r$ & $\overline{R^{2}(\%)}$ & $r$ & $\overline{R^{2}(\%)}$ & $r$ & $\overline{R^{2}(\%)}$ & $r$ & $\overline{R^{2}(\%)}$ & $r$ \\
\hline MAP & 0.9 & 0.09 & 13.0 & 0.36 & 3.3 & -0.18 & 48.6 & 0.69 & 0.1 & 0.03 \\
\hline MAT & 13.6 & $-0.37^{*}$ & 0.4 & -0.06 & 27.2 & $-0.52^{*}$ & 1.7 & -0.13 & 21.4 & $-0.45^{*}$ \\
\hline Sand & 36.7 & $-0.61^{*}$ & 40.8 & $-0.63^{*}$ & 27.7 & $-0.52^{*}$ & 88.0 & $-0.93^{*}$ & 35.7 & $-0.59^{*}$ \\
\hline Silt & 33.6 & $0.58^{*}$ & 22.1 & 0.47 & 11.3 & 0.33 & 96.0 & $0.97^{*}$ & 52.6 & $0.72^{*}$ \\
\hline Clay & 1.2 & 0.11 & 31.2 & $0.56^{*}$ & 2.4 & 0.15 & 37.0 & 0.60 & 4.8 & -0.21 \\
\hline Age & 27.0 & $0.52^{*}$ & 3.3 & 0.18 & 23.5 & 0.48 & 1.6 & 0.13 & 2.6 & -0.16 \\
\hline Altitude & 2.0 & 0.14 & 0.1 & 0.04 & 25.0 & $0.50^{*}$ & 100 & 1.0 & 14.0 & 0.37 \\
\hline $\mathrm{pH}$ & 3.7 & -0.20 & 6.2 & -0.25 & 8.2 & -0.28 & 1.7 & 0.13 & 10.8 & -0.32 \\
\hline
\end{tabular}

*Significant at $p<0.05$ (using $t$ test)

respiration (output) increased with MAT, indicating that a decrease in SOC concentration did not result from changes in either organic matter input or output, but from the balance between them. Chandra et al. (2016) had recorded a significant higher concentration of microbial biomass $\mathrm{C}$ in temperate forests having a maximum MAT range of $18-30^{\circ} \mathrm{C}$ than dry deciduous forests having $28-42{ }^{\circ} \mathrm{C}$. Liu et al. (2016) also recorded a negative correlation of SOC and MAT in different forests at separate provinces, citing lower temperatures slow down decomposition and respiration allowing SOC to accumulate.

The correlation between SOC and MAP was not significant for all tree types, as well as for different tree stands $(P>0.05)$. This result was in contrast to the results of Yost and Hartemink (2019) who reported that SOC generally increased with increasing rainfall. They found that, on average, SOC exceeded $2 \%$ in soils from cold and temperate zones, and increased to $4 \%$ when MAP was between 500 and $750 \mathrm{~mm}$, and $11 \%$ when MAP was between 800 and $1000 \mathrm{~mm}$. In the tropics, SOC was $<0.5 \%$ when MAP was between 500 and 700 $\mathrm{mm}$, and increased to $2 \%$ when MAP was between 800 and $1000 \mathrm{~mm}$. Calvode et al. (2020) also stated that MAP was the variable most predictive of SOC, followed by lithology, land use, and soil $\mathrm{pH}$.

\section{Age and altitude}

Tree age showed a significant and positive correlation with SOC for all tree types $(p<0.05)$ : C storage in the soil increased with tree age. Singh and Sharma (2007) reported greater SOC and available macronutrients in older plantations of Populus deltoides compared with younger plantations. In Gmelina arborea stand, the highest percentage of SOM with $4.78 \%$ was recorded in the oldest stand of 20 years while the least $0.7 \%$ was recorded in the youngest stand of 10 years (Adekunle et al. 2011). However, it was not significant for the different types of tree stands $(p>0.05)$. Edmondson et al. (2014) reported there was no effect of tree size on soil C storage, especially for oaks where the largest individuals were 1.6 to $2.0 \mathrm{~m} \mathrm{dbh}$. The correlation of SOC with altitude was not significant overall $(p>0.05)$; however, it was significant in the deciduous tree stands $(p<0.05)$. Shedayi et al. (2016) demonstrated that organic $C$ has a

Table 4 Mean and standard deviation for the mean annual precipitation (MAP), mean annual temperature (MAT), sand, silt, clay, age, altitude, and $\mathrm{pH}$ in evergreen and mixed tree stands

\begin{tabular}{|c|c|c|c|c|c|c|c|c|c|c|}
\hline & Evergre & & & & & Mixed & & & & \\
\hline & $\operatorname{Max}$ & Min & Mean & SD & $\bar{N}$ & Max & Min & Mean & SD & $\bar{N}$ \\
\hline MAP (mm) & 1749 & 950.0 & 1366 & 305.0 & 6 & 2542 & 73 & 1159.76 & 509.24 & 21 \\
\hline MAT $\left({ }^{\circ} \mathrm{C}\right)$ & 27.00 & 19.0 & 19.65 & 0.77 & 5 & 25.00 & 7.4 & 15.57 & 5.38 & 22 \\
\hline Sand (\%) & 92.44 & 37.29 & 74.53 & 20.51 & 5 & 71.40 & 2.6 & 32.09 & 18.38 & 14 \\
\hline Silt (\%) & 43.55 & 1.87 & 12.62 & 12.84 & 5 & 86.20 & 8.0 & 46.08 & 17.40 & 14 \\
\hline Clay (\%) & 25.69 & 5.69 & 12.44 & 7.98 & 5 & 58.30 & 5.78 & 24.31 & 14.48 & 15 \\
\hline Age (yrs.) & 600.00 & 6.0 & 161.75 & 219.12 & 4 & 400.0 & 20.0 & 81.87 & 113.42 & 7 \\
\hline Altitude (m asl) & 410 & 100 & 255 & 155 & 2 & 2650 & 157.0 & 931.18 & 519.66 & 22 \\
\hline $\mathrm{pH}$ & 7.15 & 4.08 & 5.11 & 0.77 & 12 & 6.58 & 3.3 & 5.21 & 0.93 & 17 \\
\hline
\end{tabular}


Table 5 Mean and standard deviation for the mean annual precipitation (MAP), mean annual temperature (MAT), sand, silt, clay, age, altitude, and $\mathrm{pH}$ in all types of tree species

\begin{tabular}{llllll}
\hline & Max & Min & Mean & SD & $\boldsymbol{N}$ \\
\hline MAP $(\mathrm{mm})$ & 4000.00 & 73.00 & 1210.00 & 588.00 & 74 \\
MAT $\left({ }^{\circ} \mathrm{C}\right)$ & 27.00 & -1.00 & 15.49 & 7.10 & 71 \\
Sand $(\%)$ & 94.70 & 2.60 & 43.20 & 23.74 & 50 \\
Silt (\%) & 86.20 & 1.87 & 34.89 & 19.91 & 47 \\
Clay (\%) & 60.60 & 3.50 & 23.51 & 14.31 & 51 \\
Age (yrs.) & 110.00 & 6.00 & 41.46 & 23.41 & 37 \\
Altitude (m asl) & 3440.00 & 6.00 & 1065.00 & 700.00 & 71 \\
pH & 8.33 & 3.30 & 5.23 & 1.02 & 89 \\
\hline
\end{tabular}

strong positive correlation with elevation in different types of tree stands.

\section{Texture}

The sand fraction showed a significant negative correlation with SOC for all trees (Fig. 4), as well as for the different types of tree stands (Table 3 ). The silt fraction demonstrated a significant positive correlation for all trees and for evergreen and mixed types of tree stands: the average silt fraction was highest in mixed tree stands $(46.08 \%)$ (Table 4$)$. The clay fraction did not show a significant correlation with SOC, except for in coniferous tree stands $(p<0.05)$. The negative correlation of SOC with the sand fraction has been reported previously (Tiessen \& Stewart 1983; Liu et al. 2016; Zhong et al. 2018), however, a positive correlation with the silt fraction is less well documented, although Riestra et al. (2012) reported a positive correlation of SOC content with clay plus silt content in forest soils. Loam soils are $40 \%$ sand, $40 \%$ silt, and $20 \%$ clay, which is ideal for plant growth due to the desirable characteristics of these mineral particles (Sun et al. 2019). Therefore, silt composition of up to $40 \%$ is beneficial for maintaining a stable SOC level in forest ecosystems.

Sand content is affected by soil erosion; therefore, it can be used as an indicator for evaluating soil degradation under different land-use systems (Ayele et al. 2013). There is only limited information on how physical fractions and the chemical structures of SOC relate to climate and vegetation types, especially for forest soil (Watson et al. 2000). C storage is affected by soil texture and aggregation: the highest amount of soil $\mathrm{C}$ is found in the silt- and claysized fractions, while the sand-sized fraction is low in soil C (Galeote et al. 2015). Vegetation characteristics may be a local modifier of clay content under similar climatic conditions. Therefore, the correlation between SOC concentrations and clay content may be localized and climate-dependent, and regulated by the large moisture difference that plays an essential role in driving the significant positive correlation of SOC and the clay fraction (Zhong et al. 2018).

\section{$\mathrm{pH}$}

The average $\mathrm{pH}$ of all the tree stands was 5.23 (Table 5), with the lowest $\mathrm{pH}$ found in coniferous trees (4.84) (Table 6). The correlation of SOC with $\mathrm{pH}$ was not significant; however, a negative trend was observed (Table 3). Negative relationships between SOC and soil $\mathrm{pH}$ have been found in all soil groups, except the soil group, which has the highest SOC level (Zhang et al. 2020). In general, $\mathrm{pH}$ values in the topsoil were lower because topsoil is rich in organic matter, which decomposes, leading to the production of more organic acids and thus lowering the $\mathrm{pH}$ (Hong et al. 2019).

\section{Conclusions}

This review demonstrates that variation in tree species is an important factor in the amount of SOC: mixed forest stands store more SOC than simple pure forest stands. Processes associated with individual trees, such as stem

Table 6 Mean and standard deviation for the mean annual precipitation (MAP), mean annual temperature (MAT), sand, silt, clay, age, altitude, and $\mathrm{pH}$ in coniferous and deciduous tree stands. $N=$ number of tree species

\begin{tabular}{|c|c|c|c|c|c|c|c|c|c|c|}
\hline \multirow[t]{2}{*}{ Variables } & \multicolumn{5}{|c|}{ Coniferous } & \multicolumn{5}{|c|}{ Deciduous } \\
\hline & Max & Min & Mean & SD & $\bar{N}$ & Max & Min & Mean & SD & $\bar{N}$ \\
\hline MAP (mm) & 4000 & 327 & 1550 & 805 & 21 & 1450 & 73.0 & 916.0 & 419.0 & 18 \\
\hline MAT $\left.{ }^{\left({ }^{\circ}\right.} \mathrm{C}\right)$ & 21.00 & -1.0 & 12.42 & 8.73 & 20 & 27.00 & -1.0 & 14.38 & 8.07 & 16 \\
\hline Sand (\%) & 94.70 & 5.1 & 39.26 & 26.24 & 14 & 69.87 & 5.0 & 45.59 & 20.27 & 19 \\
\hline Silt (\%) & 72.10 & 2.6 & 36.66 & 17.33 & 14 & 77.00 & 6.0 & 30.97 & 18.24 & 15 \\
\hline Clay (\%) & 48.40 & 2.7 & 24.02 & 16.23 & 14 & 60.60 & 3.5 & 25.80 & 14.67 & 18 \\
\hline Age (yrs.) & 91.00 & 7.0 & 46.35 & 19.26 & 14 & 90.00 & 8.0 & 35.10 & 24.72 & 10 \\
\hline Altitude (m asl) & 3125 & 7.0 & 1038.78 & 705.78 & 25 & 3440 & 201 & 1085.6 & 729.3 & 21 \\
\hline $\mathrm{pH}$ & 7.10 & 3.4 & 4.84 & 0.87 & 25 & 8.33 & 3.4 & 5.40 & 1.15 & 28 \\
\hline
\end{tabular}


flow and litter accumulation, can have significant effects on soil chemical properties, as well as on chemical components of litter composition (Riha et al. 1986). Coniferous tree stands have a greater SOC storage capacity than deciduous tree stands. A common variable that regulates or reduces the level of SOC could not be established for the different categories of forest stands except the sand fraction. However, the silt fraction and MAT were found to have positive and inverse relationships respectively, in all forest stands, as well as in the category of mixed forest stands. As highlighted by Mayer et al. (2020), further research is necessary to tease apart the influence of species and sites. Combining a network of common garden experiments, at greater spatial scales, could identify where and how certain tree species could be beneficial to $\mathrm{C}$ soil sequestration, and in which forms and soil layers. The important influencing factors of spatial variations in SOC concentration in different forest stands are MAT, sand, and silt fractions of soil.

\section{Abbreviations}

C: Carbon; SOC: Soil organic carbon; SIC: Soil inorganic carbon; SOM: Soil organic matter; MAT: Mean annual temperature; MAP: Mean annual precipitation; C: Coniferous; D: Deciduous; E: Evergreen; M: Mixed

\section{Acknowledgements}

The author would like to acknowledge JournalEdit (http:// www.journaledit.com) for editing and reviewing the manuscript for English language.

\section{Declaration}

The author declares that the manuscript has not been published or submitted for publication elsewhere.

\section{Author's contributions}

The author has done the review of literatures. The author(s) read and approved the final manuscript.

\section{Author's information}

The author is currently working as an Associate Professor in the Department of Environmental Science, Mizoram University, India.

\section{Funding}

Not applicable.

\section{Availability of data and materials}

Not applicable.

\section{Ethics approval and consent to participate}

Not applicable.

\section{Consent for publication}

Not applicable.

\section{Competing interests}

The author declares that she has no competing interest.

Received: 18 November 2020 Accepted: 1 January 2021

Published online: 14 January 2021

\section{References}

Adekunle UAJ, Alo AA, Adekayode FO. Yields and nutrient pools in soils cultivated with Tectona grandis and Gmelina arborea in Nigerian rainforest ecosystem. J Saudi Soc Agric Sci. 2011:127-35. https://doi.org/10.1016/j.jssas. 2011.05.001.
Ali A, Ashraf Ml, Gulzar S, Akmal M, Ahmad B. Estimation of soil carbon pools in the forests of Khyber Pakhtunkhwa Province, Pakistan. J Forest Res. 2019. https://doi.org/10.1007/s1 1676-019-01059-9.

Andivia E, Rolo V, Jonard M, Formanek P, Ponette Q. Tree species identity mediate mechanisms of top soil carbon sequestration in a Norway spruce and European beech mixed forest. Ann Forest Sci. 2016;73:437-47. https:// doi.org/10.1007/s13595-015-0536-z.

Augusto L, De Schrijver A, Vesterdal L, Smolander A, Prescott C, Ranger J. Influences of evergreen gymnosperm and deciduous angiosperm tree species on the functioning of temperate and boreal forests. Biol Rev. 2015;90:444-66.

Ayele T, Beyene S, Esayas A. Changes in land uses on soil physicochemical properties: the case of smallholders fruit-based land used systems in Arba Minch, Southern Ethiopia. Int Curr Res. 2013;5:3203-10.

Batjes NH. Harmonized soil profile data for applications at global and continental scales: updates to the WISE database. Soil Use Manage. 2016;25:124-7.

Beinroth FH, Eswaran H, Palmieri F, Reich PF. Properties, classification and management of Oxisols. In: Soil management collaborative research support program Washington; 1996. p. 1-40.

Berger TW, Neubauer C, Glatzel G. Factors controlling soil carbon and nitrogen stores in pure stands of Norway spruce (Picea abies) and mixed species stands in Austria. Forest Ecol Manag. 2002;159:3-14.

Binkley D. The influence of tree species on forest soils: processes and patterns: Special publication-Agronomy Society of New Zealand; 1995. p. 1-34.

Blonska E, Lasota J, Gruda P. Enzymatic activity and stabilization of organic matter in soil with different detritus inputs. Soil Sci Plant Nutr. 2017;63:242-7. https://doi.org/10.1080/00380768.2017.1326281.

Calvode AR, Luis E, Febrero-Bande M, Galinanes J, Macias F, Ortiz R, Casas F. Soil organic carbon in peninsular Spain: Influence of environmental factors and spatial distribution. Geoderma. 2020;370:114365. https://doi.org/10.1016/j. geoderma.2020.114365.

Chae HM, Cha S, Lee SH, Choi MJ, Shim JK. Age-related decomposition of Quercus mongolica branches. Plant Ecol. 2016;217:945-57. https://doi.org/10. 1007/s11258-016-0620-y.

Chandra LR, Gupta S, Pande V, Singh N. Impact of forest vegetation on soil characteristics: a correlation between soil biological and physic-chemical properties. 3Biotech. 2016;6:188. https://doi.org/10.1007/s13205-016-0510-y.

Chapin F III. Effects of plant traits on ecosystem and regional processes: a conceptual framework for predicting the consequences of global change. Ann Bot. 2003;91:455-63.

Chen F, Song N, Chen G, Wang J. Effects of exotic species Larix kaemferi on diversity and activity of soil microorganisms in Dalaoling National Forest Park. Ecol Proc. 2015;4:10. https://doi.org/10.1186/s13717-015-0037-x.

Day PR. Particle fractionation and particle-size analysis. In: Black CA, editor. Methods of soil analysis. Madison: American Society of Agronomy; 1965. p. 545-67.

DeVos B, Lettens S, Muys S, Muys B, Deckers JA. Walkley-Black analysis of forest soil organic carbon:recovery, limitations and uncertainty. Soil Use Manage. 2007;23:221-9.

Driessen P. Lecture notes on the major soils of the world: set 5: Cambisols. Rome: FAO; 2001. p. 1-5.

Edmondson JL, OSullivan OS, Inger R, Inger R, Potter J, McHugh N, Gaston K, Leake JR. Urban tree effects on soil organic carbon. PLoS ONE. 2014;9: e101872. https://doi.org/10.1371/journal.pone.0101872.

Eswaran H, Reich PF, Beinroth FH, Padmanabhan E, Moncharoen P. Global carbon stocks. In: Lal R, Kimble JM, Eswaran H, Stewart BA, editors. Global climate change and pedogenic carbonates. Boca Raton: Lewis Publishers; 2000. p. 15-26.

FAO, ITPS. Status of the world's soil resources-technical summary. Rome: Food and Agriculture Organization of the United Nations and Intergovernmental Technical Panel on Soils; 2015

Finzi AC, Van Breemen N, Canham CD. Canopy tree soil interactions within temperate forests: species effects on soil carbon and nitrogen. Ecol Appl. 1998:8:440-6.

Fry DL, Stevens JT, Potter AT, Collins BM, Stephens SL. Surface fuel accumulation and decomposition in old growth pine mixed conifer forests, northwestern Mexico. Fire Ecol. 2018;14:6. https://doi.org/10.1186/s42408-018-0017-5.

Galeote GMA, Trigalet S, VanWesemael B. Effect of land abandonment on soil organic carbon fractions along a Mediterranean precipitation gradient. Geoderma. 2015;249-250:69-78.

Gruba P, Socha J, Blonska E, Lasota J. Effect of variable soil texture metal saturation of soil organic matter (SOM) and tree species composition on spatial distribution of SOM in forests soils in Poland. Sci Total Environ. 2015; 521-522:90-100. 
Guedes BS, Olsson BA, Karltun E. Effects of 34 year old Pinus taeda and Eucalyptus grandis plantations on soil carbon and nutrient status in former miombo forest soils. Global Ecol Conserv. 2016;8:190-202.

Heckman K, Campbell J, Powers H, Law B, Swanston C. The influence of fire on the radiocarbon signature and character of soil organic matter in the Siskiyou national forest, Oregon, USA. Fire Ecol. 2013;9:40-57. https://doi.org/ 10.4996/fireecology.0902040.

Hong S, Gan P, Chen A. Environmental controls on soil pH in planted forest and its response to nitrogen deposition. Environ Res. 2019;172:159-65. https://doi. org/10.1016/j.envres.2019.02.020.

Huang YH, Hung CY, Lin I, Kume T, Menyailo OV, Cheng CH. Soil respiration patterns and rates at three Taiwanese forest plantations: dependence on elevation, temperature, precipitation and litterfall. Botan Stud. 2017;58:49. https://doi.org/10.1186/s40529-017-0205-7.

Ikhajeagbe B, Ogwu MC, Lawrence AE. Single-tree influence of Tectona grandis Linn. f. on plant distribution and soil characteristics in a planted forest. Bull Nat Res Centre. 2020;44:29. https://doi.org/10.1186/s42269-020-00285-0.

Jandl R, Lindner M, Vesterdal L, Bauwens B, Baritz R, Hagedorn F, Johnson DW, Minkkinen K, Byrne KA. How strongly can forest management influence soil carbon sequestration? Geoderma. 2007;137:253-68.

Janzen $\mathrm{HH}$. The soil carbon dilemma: Shall we hoard it or use it? Soil Biol Biochem. 2006:38:419-24.

Kasa G, Molla E, Abiyu A. Effects of Eucalyptus tree plantations on soil seed bank and soil physicochemical properties of Qimbaba forest. Cogent Food Agric. 2019;5:1711297. https://doi.org/10.1080/23311932.2019.1711297.

Kooijman AM, VanMourik JM, Shilder ML. The relationship between N mineralization or microbial biomass $\mathrm{N}$ with micromorphological properties in beech forest soils with different texyure and pH. Biol Fertil Soils. 2009;45:44959. https://doi.org/10.1007/s00374-009-0354-2.

Koutika LS, Nyogi S, Cafiero L, Bevivino A. Soil organic matter quality along rotations in acacia and eucalypt plantations in the Congolese Coastal Plains. Forest Ecosys. 2019;6:39. https://doi.org/10.1186/s40663-019-0197-8.

Kutsch WL, Persson T, Schrumpf M, Moyano FE, Mund M, Anderson S, Schulz ED. Heterotrophic soil respiration and soil carbon dynamics in the deciduous Hainich forest obtained by three approaches. Biogeochemistry. 2010;100: 167-83. https://doi.org/10.1007/s10533-010-9414-9.

Lal R. Soil carbon sequestration to mitigate climate change. Geoderma. 2004;123: $1-22$.

Lal R, Kimble J, Levine E, Whitman C. World soils and greenhouse effect: an overview. In: Lal R, Kimble JM, Levine E, Stewart BA, editors. Soils and global change. Boca Raton: CRC press; 1995. p. 1-7.

Lee EP, Lee SI, Jeong HM, Han YS, Lee SY, Park JH, Jang RH, Hong YS, Jung YH, Kim EJ, Lee $\mathrm{SH}$, You YH. Valuation of ecosystem services in the organic carbon of the Pinus densiflora forest at Mt. Namson, Seoul Metropolitan city. J Ecol Environ. 2019;43:35. https://doi.org/10.1186/s41610-019-0136-1.

Lin YT, Hu H, Whitman WB, Coleman DC, Chiu C. Comparison of soil bacterial communities in a natural hardwood forest and coniferous plantations in perhumid subtropical low mountains. Botan Stud. 2014;55:50. https://doi.org/ 10.1186/s40529-014-0050-x.

Liu M, Sui X, Hu Y, Feng F. Microbial community structure and the relationship with soil carbon and nitrogen in an original Korean pine forest of Changbai Mountain, China. BMC Microbiol. 2019;19:218. https://doi.org/10.1186/s12866019-1584-6.

Liu MY, Chang QR, Qi YB, Liu J, Chen T. Aggregation and soil organic carbon fractions under different landuses on the tableland of the Loess Plateau of China. Catena. 2014;115:19-28. https://doi.org/10.1016/j.catena.2013.11.002.

Liu Y, Li S, Su X, Yu X. Variations of forest soil organic carbon and its influencing factors in east China. Ann Forest Sci. 2016;73:501-11.

Lukina NV, Tikhonova EV, Danilova MA, Bakhmet ON, Kryshen AM, Tebenkova DN, Kuznetsova Al, et al. Associations between forest vegetation and the fertility of soil organic horizons in northwestern Russia. Forest Ecosys. 2019;6:34. https://doi.org/10.1186/s40663-019-0190-2.

Mandal G, Joshi SP. Analysis of vegetation dynamics and phytodiversity from three dry deciduous forests of Doon valley, Western Himalaya, India. J Asia Pac Bioderv. 2014;7:292-304. https://doi.org/10.1016/j.japb.2014.07.006.

Margesin R, Minerbi S, Schinner F. Litter decomposition at two forest sites in the Italian alps: a field study. Arctic Antarctic Alpine Res. 2016;48:127-38. https:// doi.org/10.1657/AAAR0015.012.

Marler TE, Dongeol N, Cruz GN. Leucaena leucocephala and adjacent native limestone forest habitats contrast in soil properties on Tinial island. Commun Integr Biol. 2016;9:e1212792. https://doi.org/10.1080/19420889.2016.1212792.
Matteucci M, Gruening C, Ballarin IG, Seufert G, Cescatti A. Components, drivers and temporal dynamics of ecosystem respiration in a Mediterranean pine forest. Soil Biol Biochem. 2015;88:224-35. https://doi.org/10.1016/j.soilbio.2015.05.017.

Mayer M, Prescott ECM, Abaker WEA, Augusto L, Cecillon L, Ferreira GW, et al. Tamm Review: Influence of forest management activities on soil organic carbon stocks: A knowledge synthesis. Forest Ecol Manag. 2020;466:118127. https://doi.org/10.1016/j.foreco.2020.118127.

McBratney AB, Stockmann U, Angers DA, Minasny B, Field DJ. Challenges for soil organic carbon research. In: Hartemink AE, McSweeney K, editors. Soil carbon progress in soil science. Switzerland: Springer International Publishing; 2014. p. 1-14. https://doi.org/10.1007/978-3-319-04084-4-1.

Meena A, Hanif M, Dinakaran J, Rao KS. Soil moisture controls the spatiotemporal pattern of soil respiration under different landuse systems in a semi-arid ecosystem of Delhi, India. Ecol Process. 2020;9:15. https://doi.org/ 10.1186/s13717-020-0218-0.

Miao W, LaiYe QU, KeMing MA, Xiu Y. Soil microbial properties under different vegetation types on mountain Han. Sci Chin Life Sci. 2013;56:561-70. https:// doi.org/10.1007/s11427-013-4486-0.

Miller WP, Miller MD. A micro-pipette method for soil mechanical analysis. Commun Soil Sci Plant Anal. 1987;18:1-15.

Mitchell RJ, Campbell CD, Chapman SJ, Cameron CM. The ecological engineering impact of a single tree species on the soil microbial community. J Ecol. 2010; 98:50-61.

Moslehi M, Habashi H, Khormali F, Ahmadi A, Brunner I, Zimmermann S. Base cation dynamics in rainfall, throughfall, litterflow and soil solution under Oriental beech (Fagus Orientalis Lipsky) trees in northern Iran. Ann Forest Sci. 2019;76:55. https://doi.org/10.1007/s13595-019-0837-8.

Oostra S, Majdi H, Olson M. Impact of tree species on soil carbon stocks and soil acidity in southern Sweden. Scand J Forest Res. 2006;21:354-71.

Ouyang S, Xiang W, Gou M, Lei P, Chen L, Deng X, Zhao Z. Variations in soil carbon, nitrogen, phosphorus and stoichiometry along forest succession in southern China. Biogeosci Discuss. 2017. https://doi.org/10.5194/bg-2017-408.

Owen JS, King HB, Wang MK, Sun HL. Net nitrogen mineralization and nitrification rates in forest soil in northeastern Taiwan. Soil Sci Plant Nutr. 2010;56:177-85. https://doi.org/10.1111/j.1747-0765.2009.00427.x.

Panda T. Role of fungi in litter decomposition associated with Casuarina equisetifolia L. plantations in coastal sand dunes, Orissa India. Int Biodiv Sci Ecosys Serv Manag. 2020;6:52-60. https://doi.org/10.1080/21513732.2020517443.

Riestra D, Noellemeyer E, Quiroga A. Soil texture and forest condition. The effect of afforestation on soil quality parameters. Soil Sci. 2012;177:279-87.

Riha SJ, James BR, Senesac GP, Pallant E. Spatial variability of soil pH and organic matter in forest plantations. Soil Sci Soc Am J. 1986;50:1347-52.

Saiz G, Bird MI, Domingues T, Schrodt F, Schwarz M, Feldpausch TR, Veenendaal E, Djagbletey G, Hien F, Compaore H, et al. Variation in soil carbon stocks and their determinants across a precipitation gradient in west Africa. Glob Change Biol. 2012;18:1670-83.

Shedayi AA, Xu M, Naseer I, Khan B. Altitudinal gradients of soil and vegetation carbon and nitrogen in a high altitude nature reserve of Karakoram ranges. SpringerPlus. 2016;5:320. https://doi.org/10.1186/s40064-016-1935-9.

Sheikh MA, Kumar M, Bussmann R. Altitudinal variation in soil organic carbon stock in coniferous subtropical and broadleaved temperate forests in Garhwal Himalaya. Carbon Bal Manag. 2009;4:6. https://doi.org/10.1185/17500680-4-6.

Sheng Y, Cong J, Lee H, Yang L, Liu Q, Li D, Zhang Y. Broad leaved forest types affect soil fungal community structure and soil organic carbon contents. Microbiol Open. 2019;8:e874. https://doi.org/10.1002/mbo3784.

Shi Y, Baumann F, Ma Y, Song C, Uhn PK, Scholten T, He JS. Organic and inorganic carbon in the topsoil of the Mongolian and Tibetan grasslands: pattern, control and implications. Biogeosciences. 2012;9:2287-99.

Si Y, Xiong LI, Chen Y, Zhu J, Xie J, Gao R, Yang Y. Contribution of the vertical movement of dissolved organic carbon to carbon allocation in two distinct soil types under Castanopsis fargesii Franch and C. carlesii (Hemsl.), Hayata forests. Ann Forest Sci. 2018;75:-79. https://doi.org/10.1007/s13595-018-0756-0.

Singh B, Sharma KN. Tree growth and nutrient status of soil in a Poplar (Populus deltoides Bartr.)-based agroforestry system in Punjab, India. Agroforest Sys. 2007;70:125-34.

Stanek M, Piechnik KL, Stefanowiez AM. Invasive red oak (Quercus rubra L.) modifies soil physicochemical properties and forest understory vegetation. Forest Ecol Manag. 2020;472:118253. https://doi.org/10.1016/j.foreco.2020. 118253. 
Suaze J, Jones SP, Wingate L, Wohl S, Ogee J. The role of soil pH on soil carbonic anhydrase activity. Biogeosci Discuss. 2017. https://doi.org/10.5194/bg-2017214.

Sugihara S, Fujimori Y, Shibata M, Sawada K, Tanaka H, Mvondoze AD, Araki S, Kosaki T, Funakawa S. Effects of 3-year cultivation on the soil nutrient status in a tropical forest and savanna of central Africa as determined by the microbial response to substrate addition. Soil Sci Plant Nutr. 2018;64:728-35. https://doi.org/10.1080/00380760.2018.1517585.

Sugihara S, Shibata M, Mvondoze AD, Araki S, Funakawa S. Effect of vegetation on soil C, N, P and other minerals in Oxisols at the forest savanna transitions zone of central Africa. Soil Sci Plant Nutr. 2014;60:45-59. https://doi.org/10. 1080/00380768.2013.866523

Sugihara S, Shibata M, Mvondo-Ze AD, Araki S, Kosaki T, Funakawa S. Soil phosphorus of stable fraction differentially associate with carbon in the tropical forest and savanna of eastern Cameroon. Soil Sci Plant Nutr. 2017;63: 616-27. https://doi.org/10.1080/00380768.2017.1403841.

Sun P, Zhuge Y, Zhang J, Cai Z. Soil pH was the main controlling factor of the denitrification rates and N2/N20 emission ratios in forest and grasslands soils along the Northern China Transect (NeCT). Soil Sci Plant Nutr. 2012;58:51725. https://doi.org/10.1080/00380768.2012.703609.

Sun X, Tang Z, Ryan MG, You Y, Sun OJ. Changes in soil organic carbon contents and fractionation of forests along a climatic gradient in China. Forest Ecosys. 2019;6:1. https://doi.org/10.1186/s40663-019-0161-7.

Takahashi M, Hirai K, Limtong P, Chaveevan L, Panuthai S, Suksawang S, Somchai A, Marod D. Topographic variation in heterotrophic and autotrophic soil respiration in a tropical seasonal forest in Thailand. Soil Sci Plant Nutr. 2011; 57:452-65. https://doi.org/10.1080/00380768.2011.589363.

Tellen VA, Yerima BPK. Effects of land use change on soil physicochemical properties in selected areas in the North West region of Cameroon. Environ Sys Res. 2018;7:3. https://doi.org/10.1186/s40068-018-0106-0.

Thomaz EL. Realistic soil-heating gradient temperature linearly changes most of the soil chemical properties. Soil Sci Plant Nutr. 2017;63:84-91. https://doi. org/10.1080/00380768.2016.1255538.

Tiessen H, Stewart JWB. Particle size fractions and their use in studies of soil organic matter: II cultivation effect on organic matter composition in size fractions. Soil Sci Soc Am J. 1983:47:509-14.

Vesterdal L, Clarke N, Sigurdsson BD, Gundersen P. Do tree species influence soil carbon stocks in temperate and boreal forest? Forest Ecol Manag. 2013;309: 4-18.

Vesterdal L, Schmidt IK, Callesen IC, Nilsson LO, Gundersen P. Carbon and nitrogen in forest floor and mineral soil under six common European tree species. Forest Ecol Manag. 2008;255:35-48.

Walkley A. A critical examination of a rapid method for determining organic carbon in soils: effect of variations in digestion conditions and inorganic soil constituents. Soil Sci. 1947;63:251-64.

Walter K, Don A, Tiemeyer B, Freibauer A. Determining soil bulk density for carbon stock calculations: a systematic method comparison. Soil Sci Soc Am J. 2016;80:579. https://doi.org/10.2136/ssaj2015.11.0407.

Watson RT, Noble IR, Bolin B, Ravindranath NH, Verardo DJ, Dokken DJ. Land use, land use change and forestry. Cambridge: Cambridge University Press; 2000. p. 375.

Weber OB, DaSilva MCB, DaSilva CF, DeSouza JA, Taniguch CAK, Garruti DS, Romero RE. Biological and chemical attributes of soil under forest species in Northeast Brazil. J Forest Res. 2019;31:1959-73. https://doi.org/10.1007/ s11676-019-00982-1.

Weemstra M, Sterck FJ, Visser EJW, Kyper TW, Goudzwaard L, Mommer L. Fineroot trait plasticity of beech (Fagus sylvatica) and Spruce (Picea abies) forests on two contrasting soils. Plant Soil. 2017;415:175-88. https://doi.org/10.1007/ s11104-016-3148-y.

Wyse SV. Growth responses of five forest plant species to the soils formed beneath New Zealand Kauri (Agathis australis), New Zealand. J Botany. 2012; 50:411421. https://doi.org/10.1080/0028825X.2012.724428.

Yadava PS, Devi AS. Wood and leaf litter decomposition of Dipterocarpus tuberculatus Roxb. in a tropical deciduous forest of Manipur in North-East India. Curr Sci. 2007;9:243-6.

Yam G, Tripathi OP, Das DN. Modelling of total soil carbon using readily available soil variables in temperate forest of Eastern Himalaya, Northeast India. Geol Ecol Landscapes. 2019. https://doi.org/10.1080/24749508.2019.1706295.

Yang AR, Soa Y, Noh NJ, Lee SK, Jo W, Son J, Kim C, Bae S, Lee S, Kim H, Hwang $J$. Effect of thinning on carbon storage in soil, forest floor and coarse woody debris of Pinus densiflora stands with different stand ages in Gangwon-do, central Korea. Forest Sci Tech. 2011;7:30-7. https://doi.org/10.1080/21580103. 2011.559936.

Yao X, Yu K, Deng Y, Liu J, Lai Z. Spatial variability of soil organic carbon and total nitrogen in the hilly red soil region of Southern China. J Trop Res. 2019. https://doi.org/10.1007/s11676-019-01014-8.

Yost LJ, Hartemink AE. Soil organic carbon in sandy soils: A review. In: Advances in Agronomy, vol. 158: Academic; 2019. p. 217-310. https://doi.org/10.1016/ bs.agron.2019.07.004.

You Y, Wang J, Huang X, Tang Z, Liu S, Sun OJ. Relating microbial community structure to functioning in forest soil organic carbon transformation and turnover. Ecol Evol. 2014:4:633-47.

Yu Y, Shen W, Yin Y, Zhang J, Cai Z, Zhong W. Response of soil microbial diversity to land use conversion of natural forests to plantations in a subtropical mountainous area of Southern China. Soil Sci Plant Nutr. 2012;58: 450-61. https://doi.org/10.1080/00380768.2012.708645.

Zehetgruber B, Kobler J, Dirnbock T, Jandle R, Seidl R, Schindlbacher A. Intensive ground vegetation growth mitigates the carbon loss after forest disturbance. Plant Soil. 2017;420:239-52. https://doi.org/10.1007/s11104-017-3384-9.

Zhang J, Zhang D, Jina Z, Zhou H, Zhao Y, Wei D. Litter decomposition and the degradation of recalcitrant components in Pinus massoniana plantations with various canopy densities. J Forest Res. 2019;30:1395-405. https://doi.org/10. 1007/s11676-018-0715-5.

Zhang X, Guo J, Vogt RD, Mulder J, Wang Y, Qian JW, Zhang X. Soil acidification as an additional driver to organic carbon accumulation in major Chinese croplands. Geoderma. 2020;366:114234. https://doi.org/10.1016/j.geoderma. 2020.114234.

Zhong Z, Chen Z, Xu Y, Ren C, Yang G, Han X, Ren G, Feng Y. Relationship between soil organic carbon stocks and clay content under different climatic conditions in Central China forests. Forests. 2018;9:598. https://doi.org/10. 3390/ $\$ 9100598$

Zhou L, Sun Y, Saeed S, Zhang B, Luo M. The difference of soil properties between pure and mix Chinese fir (Cunninghamia lanceolata) plantations depends on tree species. Glob Ecol Conserv. 2020;22:e01009. https://doi.org/ 10.1016/j.gecco.2020.e01009.

Zhou W, Han G, Liu M, Li X. Effects of soil pH and texture on soil carbon and nitrogen in soil profiles under different land uses in Mun River Basin, Northeast Thailand. Peer J. 2019. https://doi.org/10.7717/PeerJ.7880.

\section{Publisher's Note}

Springer Nature remains neutral with regard to jurisdictional claims in published maps and institutional affiliations.

Ready to submit your research? Choose BMC and benefit from:

- fast, convenient online submission

- thorough peer review by experienced researchers in your field

- rapid publication on acceptance

- support for research data, including large and complex data types

- gold Open Access which fosters wider collaboration and increased citations

- maximum visibility for your research: over $100 \mathrm{M}$ website views per year

At $\mathrm{BMC}$, research is always in progress.

Learn more biomedcentral.com/submissions 